Swarthmore College

Works

10-22-1999

\title{
Computational Study Of Molecular Hydrogen In Zeolite Na-A. I. Potential Energy Surfaces And Thermodynamic Separation Factors For Ortho And Para Hydrogen
}

\author{
Cherry-Rose K. Anderson , '95 \\ D. F. Coker \\ J. Eckert \\ Amy Lisa Graves \\ Swarthmore College, abug1@swarthmore.edu
}

Follow this and additional works at: https://works.swarthmore.edu/fac-physics

Part of the Physics Commons

Let us know how access to these works benefits you

\section{Recommended Citation}

Cherry-Rose K. Anderson , '95; D. F. Coker; J. Eckert; and Amy Lisa Graves. (1999). "Computational Study Of Molecular Hydrogen In Zeolite Na-A. I. Potential Energy Surfaces And Thermodynamic Separation Factors For Ortho And Para Hydrogen". Journal Of Chemical Physics. Volume 111, Issue 16. 7599-7613. DOI: $10.1063 / 1.480104$

https://works.swarthmore.edu/fac-physics/178

This work is brought to you for free by Swarthmore College Libraries' Works. It has been accepted for inclusion in Physics \& Astronomy Faculty Works by an authorized administrator of Works. For more information, please contact myworks@swarthmore.edu. 


\section{AD| $\begin{aligned} & \text { The Journal of } \\ & \text { Chemical Physics }\end{aligned}$}

Computational study of molecular hydrogen in zeolite Na-A. I. Potential energy surfaces and thermodynamic separation factors for ortho and para hydrogen

Cherry-Rose Anderson, David F. Coker, Juergen Eckert, and Amy L. R. Bug

Citation: The Journal of Chemical Physics 111, 7599 (1999); doi: 10.1063/1.480104

View online: http://dx.doi.org/10.1063/1.480104

View Table of Contents: http://scitation.aip.org/content/aip/journal/jcp/111/16?ver=pdfcov

Published by the AIP Publishing

\section{Articles you may be interested in}

Potential energy surface for interactions between two hydrogen molecules

J. Chem. Phys. 129, 094304 (2008); 10.1063/1.2975220

Computational study of molecular hydrogen in zeolite Na-A. II. Density of rotational states and inelastic neutron scattering spectra

J. Chem. Phys. 114, 10137 (2001); 10.1063/1.1352733

Hydrogen adsorption in the NaA zeolite: A comparison between numerical simulations and experiments

J. Chem. Phys. 112, 5991 (2000); 10.1063/1.481201

Ro-vibrational Stark effect on H 2 and D 2 molecules adsorbed in NaA zeolite

J. Chem. Phys. 110, 5943 (1999); 10.1063/1.478494

Analytic potential energy surfaces and their couplings for the electronically nonadiabatic chemical processes $\mathrm{Na}$ $(3 \mathrm{p})+\mathrm{H} 2 \rightarrow \mathrm{Na}(3 \mathrm{~s})+\mathrm{H} 2$ and $\mathrm{Na}(3 \mathrm{p})+\mathrm{H} 2 \rightarrow \mathrm{NaH}+\mathrm{H}$

J. Chem. Phys. 110, 4315 (1999); 10.1063/1.478314

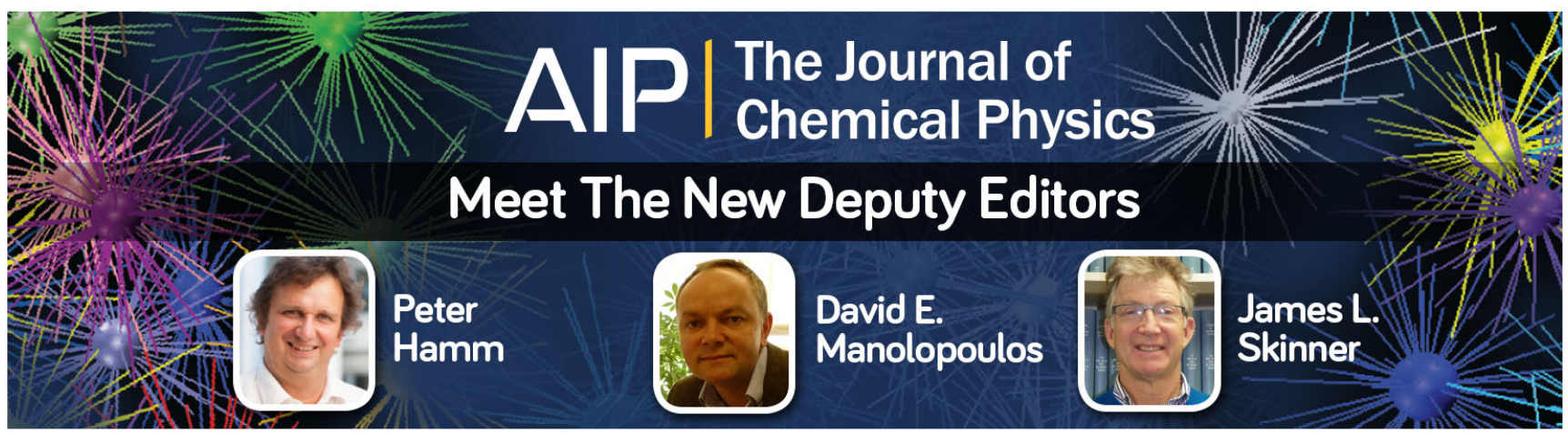




\title{
Computational study of molecular hydrogen in zeolite Na-A. I. Potential energy surfaces and thermodynamic separation factors for ortho and para hydrogen
}

\author{
Cherry-Rose Anderson \\ Department of Physics and Astronomy, Swarthmore College, Swarthmore, Pennsylvania 19081 \\ David F. Coker \\ Department of Chemistry, Boston University, Boston, Massachusetts 02215 \\ Juergen Eckert \\ Los Alamos National Laboratory, Los Alamos, New Mexico 87545 \\ Amy L. R. Bug ${ }^{\text {a) }}$ \\ Department of Physics and Astronomy, Swarthmore College, Swarthmore, Pennsylvania 19081
}

(Received 12 March 1999; accepted 21 July 1999)

\begin{abstract}
We simulate $\mathrm{H}_{2}$ adsorbed within zeolite Na-A. We use a block Lanczos procedure to generate the first several (9) rotational eigenstates of the molecule, which is modeled as a rigid, quantum rotor with an anisotropic polarizability and quadrupole moment. The rotor interacts with Na cations and $\mathrm{O}$ anions; interaction parameters are chosen semiempirically and the truncation of electrostatic fields is handled with a switching function. A Monte Carlo proceedure is used to sample a set of states based on the canonical distribution. Potential energy surfaces, favorable adsorbtion sites, and distributions of barriers to rotation are analyzed. Separation factors for ortho-parahydrogen are calculated; at low temperatures, these are controlled by the ease of rotational tunneling through barriers. () 1999 American Institute of Physics. [S0021-9606(99)70139-X]
\end{abstract}

\section{INTRODUCTION}

Zeolites $^{1}$ are aluminosilicates which contain rigid and regular systems of cavities and channels with dimensions ranging from around $3 \AA$ to well over $10 \AA$. These characteristics give rise to their ability to retain and filter molecules with a high degree of selectivity, ${ }^{2,3}$ and hence their use as molecular "sieves." Aluminosilicates also contain chargebalancing entities, due to the negative charge of the $\mathrm{AlO}_{4}$ tetrahedra which alternate with $\mathrm{SiO}_{4}$ tetrahedra in the framework. These entities are either acid sites ( $-\mathrm{OH}$ groups), or cations located in extra-framework sites. These sites give rise to the catalytic activity of zeolites, which via their geometries, polarity, and the identity and location of acidic sites or ions, can dominate the dynamics of chemical reactions between adsorbed reagents. ${ }^{1,3,4}$ These same properties also give rise to the utility of zeolites in gas separation, and their potential as storage media for gaseous fuels like methane and hydrogen. ${ }^{5}$

Despite the fact that zeolites have been used on an industrial scale for many decades, detailed atomic-level information on the interaction of zeolite hosts with adsorbate molecules has only recently begun to emerge. Much of this progress has been accomplished by a close interplay of experimental investigations with various types of theoretical calculations and simulations. This paper, and one that follows, ${ }^{6}$ concern a simulation of molecular hydrogen, a

\footnotetext{
a) Author to whom all correspondence should be addressed; electronic mail: abug1@swarthmore.edu
}

guest molecule with a role in all of the applications discussed above. Hydrogen may be viewed as one of the most versatile probes of adsorption sites in zeolites, on account of its small size and weak interaction with the host. $\mathrm{H}_{2}$, as well as other diatomics, have been used as probes of catalytic acid sites, ${ }^{7}$ and $\mathrm{H}_{2}$ may probe dual acid-base sites as well. ${ }^{8}$

One of the applications of zeolites in the area of separation has been their use to separate ortho- from parahydrogen. This is based on the different adsorption strengths of the two rotational states of the hydrogen molecule. The experimental efficiency with which ortho- and parahydrogen can be separated, the so-called separation factor, is therefore a good benchmark for interatomic potentials used in theoretical calculations of this quantity. In the current paper, we will present a Monte Carlo (MC) simulation of adsorbed $\mathrm{H}_{2}$ in the zeolite Na-A, with realistic guest-host interaction potentials and a quantum mechanical treatment of the rotations of the $\mathrm{H}_{2}$ molecule. A MC sampling proceedure will produce a set of center-of-mass positions in the zeolite which are consistent with the canonical distribution at a given temperature. Potential energy surfaces encountered by the hindered $\mathrm{H}_{2}$ rotor at these positions will be described, and separation factors will be computed for comparison with experimental data. The second part of our work ${ }^{6}$ will examine the rotational wave functions of the hindered rotor, and present direct measurements of the ortho- to parahydrogen rotational tunneling transition by inelastic neutron scattering. Experimental data will be compared with computed rotational potential barriers, and with a computed neutron spectrum. 


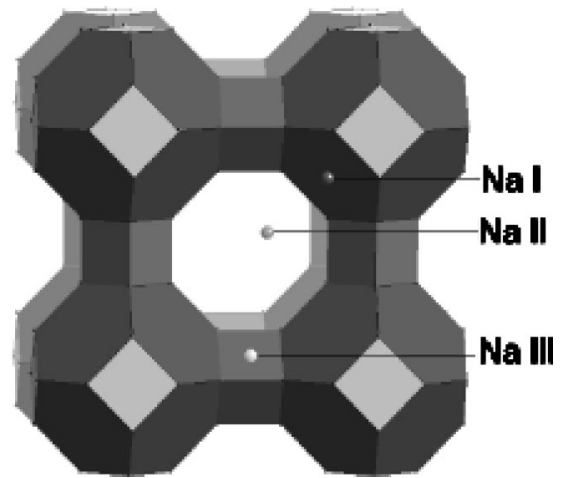

FIG. 1. Zeolite Na-A (also referred to as 4A).

\section{MODEL AND METHODS}

\section{A. Guest-host interactions}

The unit cell formula of dehydrated zeolite Na-A (also known as $4 \mathrm{~A}$ ) is $\mathrm{Na}_{12} \mathrm{Al}_{12} \mathrm{Si}_{12} \mathrm{O}_{48}$. (There are an additional $27 \mathrm{H}_{2} \mathrm{O}$ in the hydrated form. $)^{9}$ The crystal is cubic with unit cell diameter $12.3 \AA$, and space group Pm $3 \mathrm{~m}$ if one does not distinguish between $\mathrm{Si}$ and $\mathrm{Al}$. With the ratio of $\mathrm{Si} / \mathrm{Al}$ equal to unity, $\mathrm{Si}$ and $\mathrm{Al}$ alternate in the lattice (Loewenstein's rule). Additional disorder arises from the positions of a subset of $\mathrm{Na}$ cations. ${ }^{10,11}$ At room temperature and below, each of three (termed $\mathrm{Na}_{\text {II }}$ ) cations will choose one of four roughly degenerate local minima in the plane of an eight ring (see Fig. 1). One $\left(\mathrm{Na}_{\text {III }}\right)$ will choose one of twelve roughly degenerate local minima within the supercage, near a four ring. Our simulation does not distinguish between $\mathrm{Si}$ and $\mathrm{Al}$ and, as is common in the guest-zeolite literature, treats the $\mathrm{Si}_{1 / 2} \mathrm{O}$ group as a single center of force. ${ }^{12} \mathrm{Na}$ cations are placed in their crystallographic positions, chosen arbitrarily in the case of $\mathrm{Na}_{\text {II }}$ and $\mathrm{Na}_{\mathrm{III}}$. (In contrast, a few studies on the thermodynamics of adsorbtion place fractional charges at all sites with the correct symmetry. ${ }^{13}$ ) The coordinates of the atoms in a unit cell of our model zeolite can be inferred from the positions in Table I.

The potential energy for the $\mathrm{H}_{2}$ guest is a sum of terms:

$$
U=U_{\text {disp }}+U_{\text {rep }}+U_{\text {pol }}+U_{\text {multipole }} \text {. }
$$

The first two are atom-atom terms, a (London) dispersion attraction, and a short-ranged repulsion; the second two terms represent interaction with the electric field in the cavity. The electric field is imagined to stem from partial charges on $\mathrm{Na}$ and $\mathrm{O}$ atoms. While several studies proceed by simply assigning the nominal charge of $q_{\mathrm{Na}}=+1 e$ to $\mathrm{Na}$,

TABLE I. Zeolitic positions (尺̊).

\begin{tabular}{llll}
\hline \hline Atom & $x$ & $y$ & $z$ \\
\hline $\mathrm{Si} / \mathrm{Al}$ & 1.57 & 3.89 & 6.15 \\
$\mathrm{O}_{\mathrm{I}}$ & 0.0 & 3.35 & 6.15 \\
$\mathrm{O}_{\text {II }}$ & 2.56 & 2.56 & 6.15 \\
$\mathrm{O}_{\text {III }}$ & 1.94 & 4.77 & 4.77 \\
$\mathrm{Na}_{\text {I }}$ & 3.67 & 3.67 & 3.67 \\
$\mathrm{Na}_{\text {II }}$ & 0.86 & 0.86 & 6.15 \\
$\mathrm{Na}_{\text {III }}$ & 0.0 & -3.31 & 3.31 \\
\hline \hline
\end{tabular}

and hence $q_{\mathrm{O}}=-0.25 e$ to $\mathrm{O},{ }^{14,15}$ this tends to, for example, overestimate the strength of the electric field in the cavities, as one can infer from induced infrared bands of $\mathrm{H}_{2}$, and other zeolitic guests. ${ }^{7,15,16,17}$ The majority of our data were generated with $q_{\mathrm{Na}}=0.6 e, q_{\mathrm{O}}=-0.15 e$, consistent with the range for $q_{\mathrm{Na}}$ of $0.55-0.7 e$ that can be seen in various published simulations. ${ }^{18-21}$ Charge parameters for such studies are typically derived from quantum chemical calculations; alternatively ${ }^{20,22} \mathrm{Na}$ partial charges can be optimized by comparing the resulting normal mode frequencies to IR data. A recent high-resolution x-ray diffraction study deduced atomic charges from electronic densities, for the zeolite natrolite. ${ }^{23}$

We take

$$
\begin{aligned}
& U_{\mathrm{pol}}=-\frac{1}{2} \mathbf{E}(\mathbf{r}) \cdot \widetilde{\alpha}(\mathbf{\Omega}) \cdot \mathbf{E}(\mathbf{r}), \\
& U_{\text {multipole }}=-\frac{1}{6} \sum_{i j=1,3} Q_{i j}(\boldsymbol{\Omega}) \frac{\partial \mathbf{E}_{j}(\mathbf{r})}{\partial x_{i}},
\end{aligned}
$$

where the indices $i, j$ run over three Cartesian coordinates. $\mathbf{E}(\mathbf{r})$ is the electric field at the $\mathrm{H}_{2}$ center-of-mass location $\mathbf{r}$, $\widetilde{\alpha}(\boldsymbol{\Omega})$ is its molecular polarizability tensor, and $\widetilde{Q}(\boldsymbol{\Omega})$ is its quadrupole tensor. $\boldsymbol{\Omega}$ is a steric angle giving the orientation of the $\mathrm{H}_{2}$ bond axis, referred to Cartesian axes fixed within the zeolite. When the molecular axis points in the $z$ direction, the tensors are diagonal:

$$
\widetilde{\alpha}=\left(\begin{array}{ccc}
\alpha_{1} & 0 & 0 \\
0 & \alpha_{1} & 0 \\
0 & 0 & \alpha_{2}
\end{array}\right), \quad \widetilde{Q}=\left(\begin{array}{ccc}
\frac{-Q}{2} & 0 & 0 \\
0 & \frac{-Q}{2} & 0 \\
0 & 0 & Q
\end{array}\right)
$$

with $\alpha_{1,2}=0.71,0.93 \AA^{3}$ and $Q=0.26 e \AA^{2}{ }^{24}$ The rotation of these tensors through the angle $\boldsymbol{\Omega} \equiv(\theta, \phi)$ is accomplished in a standard way:

$$
\begin{aligned}
& \widetilde{\alpha}(\Omega)=\widetilde{R}^{T} \widetilde{\alpha} \widetilde{R}, \\
& \widetilde{R}=\left(\begin{array}{ccc}
\cos \theta \cos \phi & \cos \theta \sin \phi & -\sin \theta \\
-\sin \phi & \cos \phi & 0 \\
\sin \theta \cos \phi & \sin \theta \sin \phi & \cos \theta
\end{array}\right) .
\end{aligned}
$$

Though ones simulation results "rest heavily upon the reliability of the potentials and their parametrizations", 25 the unfortunate reality is that there is a great deal of freedom in how one can choose semiempirical potentials. Our dispersion attraction is modeled with a dipole-induced dipole term: ${ }^{26}$

$$
U_{\mathrm{dis}}=\sum_{k} \frac{-A_{k}}{r^{6}},
$$

where $k$ indexes $\mathrm{Na}$ and $\mathrm{O}$. If $A_{k}$ is calculated using a Kirkwood-Muller form ${ }^{27}$ with atomic parameters for isotropic polarizability, $\alpha$, and diamagnetic susceptibility, $\chi$, of Table II, the results, in round figures, are: $A_{\mathrm{Na}}$ $=80 \AA^{6} \mathrm{kcal} / \mathrm{mol}, \quad A_{\mathrm{O}}=390 \AA^{6} \mathrm{kcal} / \mathrm{mol}$. (A SlaterKirkwood parametrization ${ }^{27}$ with the number of $\mathrm{Na}$ electrons set to 10.4 and number of $\mathrm{O}$ electrons set to 8.15 yields roughly the same results.) A London calculation ${ }^{28}$ gives 
TABLE II. Simulation parameters.

\begin{tabular}{ccccc}
\hline \hline Species & $\alpha, 10^{-24} \mathrm{~cm}^{3}$ & $\chi, 10^{-30} \mathrm{~cm}^{3}$ & $r_{\mathrm{vdw}}, \AA$ & $I(\mathrm{kcal} / \mathrm{mol})$ \\
\hline $\mathrm{H}_{2}$ & $0.79^{\mathrm{b}}$ & $6.6^{\mathrm{h}}$ & $1.45^{\mathrm{c}, \mathrm{e}}$ & $356^{\mathrm{h}}$ \\
$\mathrm{Na}$ & $0.22^{\mathrm{a}}$ & $6.95^{\mathrm{a}}$ & $0.98^{\mathrm{a}, \mathrm{g}}$ & $512^{\mathrm{i}}$ \\
$\mathrm{O}$ & $1.4^{\mathrm{d}}$ & $17.7^{\mathrm{a}, \mathrm{d}}$ & $1.45^{\mathrm{d}, \mathrm{f}, \mathrm{g}}$ & $195^{\mathrm{i}}$ \\
\hline
\end{tabular}

${ }^{\mathrm{a}}$ Reference 14

${ }^{\mathrm{b}}$ Reference 83 .

${ }^{\mathrm{c}}$ Reference 84.

${ }^{\mathrm{d}}$ Reference 85 .

${ }^{\text {e}}$ Reference 86 .

smaller values: $\quad A_{\mathrm{Na}}=50 \quad \AA^{6} \mathrm{kcal} / \mathrm{mol}, \quad A_{\mathrm{O}}=210$ $\AA^{6} \mathrm{kcal} / \mathrm{mol}{ }^{29}$ The results of the present work correspond to the values $A_{\mathrm{Na}}=50$ and $A_{\mathrm{O}}=230 \AA^{6} \mathrm{kcal} / \mathrm{mol}$. Most relevent, the major results reported in this paper are not very sensitive to the distinction between these values (some supporting data appear in Ref. 6).

We model the short-ranged repulsion as

$$
U_{\mathrm{rep}}=\sum_{k} \frac{B_{k}(\gamma)}{r^{12}}
$$

This term is designed to make the potential energy between $\mathrm{H}_{2}$ and a single zeolite atom a minimum when they are separated by a distance corresponding to the van der Waals diameter, $r_{0}(\gamma)$, where $\gamma$ is the angle between the bond axis of $\mathrm{H}_{2}$ and the vector from $\mathrm{H}_{2}$ to the zeolite atom. We have adopted the parametrization:

$$
r_{0}(\gamma)=r_{0}^{1}+\left(r_{0}^{2}-r_{0}^{1}\right) \cos ^{2} \gamma
$$

There are various experimental and theoretical values to choose from for van der Waals radii, $r_{\mathrm{vdw}}$. With values as in Table II, we have $r_{0}^{1,2}=2.39,2.50 \AA$ for $\mathrm{Na}$ and $r_{0}^{1,2}$ $=2.86,2.97 \AA$ for $\mathrm{O}$. These are based on $\mathrm{H}_{2}$ having short and long dimensions of 2.82 and $3.04 \AA$, respectively; in line with calculations and collisional experiments. ${ }^{24,30}$ For a single zeolite atom of type $k$ at distance $r$ with $\mathrm{H}_{2}$ inclined at angle $\gamma$, the electrostatic energies of Eq. (2) are

$$
\begin{aligned}
& U_{\mathrm{pol}}=\frac{-q_{k}^{2}}{2 r^{4}}\left(\alpha_{1}+\left(\alpha_{2}-\alpha_{1}\right) \cos ^{2} \gamma\right) \equiv \frac{-q_{k}^{2}}{2 r^{4}} \alpha(\gamma), \\
& U_{\text {multipole }}=\frac{Q q_{k}}{4 r^{3}}\left(3 \cos ^{2} \gamma-1\right) \equiv \frac{q_{k}}{4 r^{3}} Q(\gamma) .
\end{aligned}
$$

In order to find $B_{k}(\gamma)$, Eqs. (5), (8), and (9) imply that we set

$$
\begin{aligned}
\frac{\partial}{\partial r}\left(\frac{B_{k}(\gamma)}{r^{12}}-\frac{A_{k}}{r^{6}}-\frac{q_{k}^{2}}{2 r^{4}} \alpha(\gamma)+\frac{q_{k}}{4 r^{3}} Q(\gamma)\right) & =0 \\
\text { at } r & =r_{0}(\gamma) .
\end{aligned}
$$

$$
B_{k}(\gamma)=\frac{r_{0}(\gamma)^{6}}{2}\left(A_{k}+\frac{q_{k}^{2} r_{0}(\gamma)^{2} \alpha(\gamma)}{3}-\frac{q_{k} r_{0}(\gamma)^{3} Q(\gamma)}{8}\right)
$$

with $r_{0}$ as in Eq. (7).

A somewhat different parametrization for the case of $\mathrm{H}_{2}-\mathrm{Na}^{+}$interactions has been made based on semiempirical Hartree-Fock (HF) calculations. ${ }^{31,32}$ Fits of the potential to an analytic form indicated that terms which are higher than second order in the angle (e.g., hexadecapole interactions) were not major contributors below a separation of about 2.5 $\AA$. It was also found, for example, that at separations greater than $6 \AA$, electrostatic, inductive terms reproduced the HF potential extremely well. While it is not obvious how parameters should compare, given the difference in the charge state of the $\mathrm{Na}$ for which we seek a potential model, Falcetta et al. found that the minimum intermolecular separation was $R$ $=2.475 \AA$ and the well had a depth of $2.95 \mathrm{kcal} / \mathrm{mol}$. (These numbers have been calculated in a variety of other quantum chemical studies, some of which are cited in Sec. IV.) These are to be compared with $R=2.4 \AA$ and $1.73 \mathrm{kcal} / \mathrm{mol}$ for our model, when $\mathrm{H}_{2}$ interacts with an isolated sodium for which $q_{\mathrm{Na}}=0.6$. (In our model, this well depth increases to 2.5 $\mathrm{kcal} / \mathrm{mol}$ when the charge is increased to $q_{\mathrm{Na}}=1.0$, but other parameters are left unchanged.) Our short range repulsive energy has a value of $86 \mathrm{kcal} / \mathrm{mol}$ at $R=1.6 \AA$, which is higher than the value of $25 \mathrm{kcal} / \mathrm{mol}$ found in Ref. 31, albeit lower than a value of $114 \mathrm{kcal} / \mathrm{mol}$ that arises from a fit to an inelastic scattering experiment. ${ }^{33}$ It might also be worthwhile to note that quantum chemical studies have taught us that the binding of $\mathrm{H}_{2}$ to an isolated $\mathrm{Na}^{+}$is almost entirely electrostatic in origin. There is a very small contribution of correlation energy to the binding energy, ${ }^{31,34,35}$ and the sodium polarizes very little. These are not true for $\mathrm{H}_{2}$ binding to some of the other metals studied. ${ }^{35}$

A cubic simulation cell of width $r_{c}=24.6 \AA$ (two unit cells) was used. Periodic boundary conditions were employed, which necessitated cutting off interaction potentials at a range of $r_{c} / 2$ or less. In the case of $U_{\text {rep }}$, a cutoff of 6.0 $\AA$ was found to be adequate. ${ }^{36}$ Since the sum in Eq. (6) had to be evaluated for all zeolite atoms, for all angles $\gamma$ on an angular grid (described below) this cutoff gave meaningful savings in computation time.

The long range electrostatic interactions necessitated a more careful treatment, though not as careful (Ewald sums) as would be required for a guest with a net charge. We employed a "switching" function which imposed an exponential drop-off on the electric field outside of a given radius $r_{1}$, and set the field outside of a second radius, $r_{2}$, to zero. Thus, the contribution to the electric field at $\mathrm{H}_{2}$ from charge $q_{k}$ at a distance $r_{k}$ became

$$
E=\frac{q_{k}}{r_{k}^{2}} S\left(r_{k}\right)
$$

where

$$
S(r)=\left\{\begin{array}{l}
0 \quad r \geqslant r_{2} \\
e^{-\left(r-r_{1}\right)^{2}} \quad r_{2}>r \geqslant_{1} . . \\
1 \quad r_{1}>r
\end{array}\right.
$$


Cutoff values of $r_{1}=r_{c} / 3$ and $r_{2}=r_{c} / 2$ were adopted based on molecular dynamics simulations of polarizable guests. ${ }^{22}$

Throughout these calculations we have neglected the influence of phonons and used a rigid zeolite lattice. There is a possibility that this approximation may have an effect on dynamical properties like the actual rate of diffusion of $\mathrm{H}_{2}$ through the zeolite. Phonon vibrations of the lattice may thus effect the passage of the $\mathrm{H}_{2}$ molecule from one cage to the next. If the equilibrium properties are dominated by time spent fluctuating within the cages, rather than passing between them, then ignoring the effects of phonons on quantities like the ratios of rotational partition functions is probably very reasonable. In the literature on dynamical simulations of guests in zeolites, the question of whether one may save computer time by using a rigid lattice is pertinent. Often the use of a rigid lattice is based on the intuition that the dynamical behavior of fairly small guests will not be altered if a flexible framework is used. ${ }^{37}$ In one study by Demontis et al. ${ }^{38}$ both flexible and rigid frameworks were used to evaluate the diffusion rate of methane in silicalite. It was found that properties like diffusion constants, activation energies, and radial distribution functions were almost unaffected by framework vibrations.

\section{B. Monte Carlo simulation}

Our goal is to find sites of adsorption, and separation factors (see Sec. III) for hydrogen spin isotopes. In a sequel to this paper, ${ }^{6}$ we will present data on molecular orientation, densities of energy states, and the resulting neutron spectra. Our method presumes that, as in Sec. II A, the energy of hydrogen at a location $\mathbf{r}$ in the crystal may be separated into a center-of-mass and an orientational term:

$$
V=V_{0}(\mathbf{r})+V_{\mathbf{r}}(\boldsymbol{\Omega}) .
$$

The first term of Eq. (1), $U_{\text {disp }}$, corresponds to $V_{0}(\mathbf{r})$; the remaining terms constitute $V_{\mathbf{r}}(\boldsymbol{\Omega})$. We further assume that the hydrogenic wave function may be represented as a product of a center-of-mass and an orientational term:

$$
\Psi=\Psi_{0}(\mathbf{r}) \phi_{\mathbf{r}}(\boldsymbol{\Omega}) .
$$

The assumption here is that the angular Schrödinger equation can be solved for each position $\mathbf{r}$; an adiabatic approximation. It is not obvious that this is appropriate, that center-ofmass degrees of freedom decouple from rotational degrees of freedom. However, one may make an approximation similar to the Born-Oppenheimer approximation, which is frequently used for electronic and nuclear motions, or molecular rotations and vibrations. The argument is given in the Appendix. There, we show that if the energy difference between rotational states is large relative to certain matrix elements connecting different states, the adiabatic approximation is satisfactory.

A related assumption in our model is that the center-ofmass "motion" of the molecule can be treated classically:

$$
\Psi_{0}(\mathbf{r})=\delta(\mathbf{r}-\mathbf{r}(\mathbf{t})) .
$$

In the current work, we do not report on the vibrational levels of the molecule as a whole in the zeolitic host. Were one to attempt this, it would probably be necessary to amend this assumption at temperatures significantly below room temperature, because quantization of vibrational levels could not be neglected, and/or because the zeolitic potential varies significantly over the de Broglie wavelength of a free hydrogen molecule.

In order to solve the Schrödinger equation for the rotational motion of hydrogen:

$$
\left(-\frac{\hbar^{2}}{2 I} \hat{L}^{2}+V_{\mathbf{r}}(\boldsymbol{\Omega})\right) \phi_{\mathbf{r}}^{k}=\epsilon_{\mathbf{r}}^{k} \phi_{\mathbf{r}}^{k},
$$

we employ a block Lanczos method. ${ }^{39}$ This is a spectral technique which seeks to diagonalize the operator

$$
\hat{W} \equiv e^{-\tau \hat{H}} .
$$

In our case, and in the limit of $\tau$ small,

$$
\hat{W} \approx e^{-\tau \hat{V} / 2} \exp \left(-\tau \frac{\hat{L}^{2}}{2 I}\right) e^{-\tau \hat{V} / 2}
$$

The eigenvectors of $\hat{W}$ in Eq. (18) approximate the eigenstates $\phi_{\mathbf{r}}^{k}$ with eigenvalues $e^{-\tau \epsilon_{\mathbf{r}}^{k}}$.

The block Lanzcos method is a general algorithm for efficiently computing a limited set of eigenvectors and eigenvalues of a large matrix $\mathbf{A}$ which requires only a procedure for operating the matrix on some arbitrary vector $\mathbf{X}$, and a standard small matrix eigensystem solver. We would like to diagonalize the Hamiltonian matrix $\mathbf{H}$ and our technique relies on the fact that the eigenvectors of $\mathbf{H}$ and $\mathbf{W}$ $=\exp [-\tau \mathbf{H}]$ are identical.

With this approach we first select a block of initial guess vectors which approximate the eigenvectors we are interested in computing accurately. Say this initial block is the set of vectors $\left\{\mathbf{Y}_{k}^{(0)}\right\}$, and we choose them to be orthogonal to one another, and normalized. Next we operate with $\mathbf{W}$ on each of the initial vectors to produce a new block of vectors $\left\{\mathbf{W} \mathbf{Y}_{k}^{(0)}=\mathbf{X}_{k}^{(1)}\right\}$. Gram-Schmidt orthogonalization is used to modify these new vectors so that they are orthogonal to one another, orthogonal to all the vectors in the previous block, and normalized. Let this new block of orthogonalized vectors be $\left\{\mathbf{Y}_{k}^{(1)}\right\}$. We now repeat this procedure involving application of $\mathbf{W}$ to the new block, and orthonormalize the vectors thus generated with respect to themselves and to all previous blocks.

It is easily shown that, because of the blockwise orthonormalization at each basis set generation cycle, the series of blocks of orthonormal vectors $\left(\mathbf{Y}^{(0)}, \mathbf{Y}^{(1)}, \mathbf{Y}^{(2)}, \ldots, \mathbf{Y}^{(n)}\right)$ forms a basis set in which the $\mathbf{W}$ matrix is block tridiagonal with nonzero elements $\mathbf{Y}_{l}^{(i) T} \mathbf{W} \mathbf{Y}_{m}^{(j)}$, where $j=i$, or $j=i$ \pm 1 . This small, sparse matrix can now be diagonalized using standard methods to give a small group of accurate eigenvectors of the much larger $\mathbf{W}$ matrix.

Precisely how many times this block generation cycle needs to be repeated depends on how well the initial vectors have been chosen. Obviously if the starting vectors were exactly the required eigenvectors, then applying $\mathbf{W}$ to them and normalizing would recover the same set of vectors, and the matrix formed using this very first block of basis vectors would already be diagonal. However, if the initial guess vec- 
tors were some approximate set, each could be written as a linear combination of the complete set of true eigenvectors. Repeated application of $\mathbf{W}$ to these initial guess vectors written in terms of their superpositions of eigenvectors would result in the coefficients of each component eigenvector being multiplied by higher and higher powers of its eigenvalue. With this approach each newly generated basis vector would look more and more like the higher eigenvalue component, and relatively less and less like the lower eigenvalue components, contained in its initial expansion. Because we seek the lowest lying energy eigenvalues, the largest eigenvalues of $\mathbf{W}$ are precisely what we would like to calculate.

The iterative Lanzcos basis set generation procedure thus disperses the different eigenvector components of the initial basis set according to their eigenvalues. Eventually, when the newly generated basis vectors are sufficiently enhanced in their higher lying eigenvalue components, they will in principle provide a good representation of the difference between the desired eigenvectors and the initial guesses. Application of a standard eigensystem solver with the basis set generated in this way will thus be able to resolve out the desired components.

As described above, the method might still require the generation of large numbers of basis vector blocks if the initial vectors are poorly chosen approximations to the vectors of interest. To overcome this problem, the method we employ $^{39,40}$ couples the block Lanzcos procedure described above with a preconditioning-relaxation approach. The idea is that if we are interested in a set of low lying eigenstates of $\mathbf{H}$, then repeated application of $\exp [-\tau \mathbf{H}]$ to our initial basis vectors will exponentially damp out the high energyeigenvalue states, enhancing the newly generated basis vectors in the low-lying energy states of interest. The larger the value of $\tau$ employed, the faster the high eigenvalue components will be relaxed out of the basis set. The Trotter factorization in Eq. (18) is, of course, only an accurate representation of $\exp [-\tau \mathbf{H}]$ in the small $\tau$ limit. The approach $^{40}$ thus merely preconditions the basis set in a series of steps starting with large $\tau$ to quickly remove the high-lying components, and then uses the resulting states as the starting basis set for more accurate, small $\tau$ calculations. For most of the calculations that we will report here, $\tau$ was lowered from 1000 a.u. in four or five steps. Block diagonalization then proceeded with a value of $\tau$ in the viscinity of 20 a.u.

In our application, the eigenfunctions of the rotational Hamiltonian are represented on a grid of $N$ points in orientational space. The values of our orientational basis functions computed on this grid of points $\left(\theta_{i}, \phi_{i}\right)$ form vectors of length $N . \hat{H}$ and $\hat{W}$ are $N \times N$ matrices. The action of these matrices is readily computed using the spherical harmonic functions and Gauss-Legendre quadrature as described below.

In order to diagonalize $\hat{W}$, one needs to evaluate quantities like

$$
\begin{aligned}
\left\langle f_{i}|\hat{W}| f_{j}\right\rangle= & \int\left\langle f_{i} \mid \mathbf{\Omega}\right\rangle e^{-\tau V_{\mathbf{r}}(\boldsymbol{\Omega}) / 2} \exp \left(-\tau \frac{\hat{L}^{2}}{2 I}\right) \\
& \times e^{-\tau V_{\mathbf{r}}(\boldsymbol{\Omega}) / 2}\left\langle\boldsymbol{\Omega} \mid f_{j}\right\rangle d \boldsymbol{\Omega}
\end{aligned}
$$

for basis functions $\left\langle\boldsymbol{\Omega} \mid f_{i}\right\rangle$. This is achieved by performing an integral transform:

$$
\begin{aligned}
\left\langle g_{i}\left|\exp \left(-\tau \frac{\hat{L}^{2}}{2 I}\right)\right| g_{j}\right\rangle & \\
= & \sum_{\substack{l=0 \\
m=-l, l}}^{\infty}\left\langle g_{i} \mid \operatorname{lm}\right\rangle \exp \left(-\tau \frac{l(l+1)}{2 I}\right)\left\langle\operatorname{lm} \mid g_{j}\right\rangle,
\end{aligned}
$$

where

$$
\left\langle l m \mid g_{j}\right\rangle=\int Y_{l}^{m}\left(\mathbf{\Omega}^{\prime}\right) g_{j}\left(\mathbf{\Omega}^{\prime}\right) d \mathbf{\Omega}^{\prime} .
$$

The transform of the function $g_{j}=e^{-\tau \hat{V} / 2} f_{j}$ has as its kernel a spherical harmonic. The integrals in Eqs. (19) and (20) were performed with Gaussian-Legendre quadrature. The space of angles $\theta, \phi$ was discretized with an angular grid of size $32 \times 32$. The forward and backward discrete transformations between the spaces of $l, m$, and $\boldsymbol{\Omega}$ are analogous to the discrete Fourier transforms (Fast Fourier transforms) between momentum and coordinate space that one might use to implement a Lanczos or other spectral method in Cartesian coordinates.

We followed $N_{\text {states }}=9$ rotational eigenstates for the current study. In order to test parameters of the algorithm, we compared with the expected eigenenergies for a free rotor, and for a hindered rotor with

$$
V(\theta)=10 \times B_{0} \sin ^{2}(\theta),
$$

whose eigenspectrum was calculated with high precision by Curl et al. ${ }^{41}$ The internuclear distance $d_{0}$, in terms of which $I=m / 2 d_{0}^{2}$, was taken as $0.77 \AA$. This value is consistent with earlier work by one of us, ${ }^{42}$ though larger the distance at which the (theoretical and experimental) interatomic potential has its minimum $(0.74 \AA),{ }^{30}$ and also slightly longer than a rigid rotor with the experimental rotational constant of $B_{0}$ $=85.4 \mathrm{~K}$. We used anywhere from two to five blocks. Our original guess vectors were the free-rotor eigenstates of hydrogen. (In a lightly perturbed system, it would be necessary to add some small amount of noise to these to remove the degeneracy. In the strongly perturbing environment of the zeolite electric field, this preconditioning was not necessary.) Using the parameters that we have mentioned above, our energies were correct to four significant figures for the two test systems.

The center of mass performs a random walk through the host, via a standard METROPOLIS MC algorithm. ${ }^{43}$ From a location $\mathbf{r}$, a trial step to a new location $\mathbf{r}^{\prime}$ is made, where $\mathbf{r}^{\prime}$ is chosen from a uniform distribution of locations centered on $\mathbf{r}$. This move is accepted or rejected, based on the ratio of partition functions $Q(\mathbf{r}) / Q\left(\mathbf{r}^{\prime}\right)$, where

$$
Q(\mathbf{r})=e^{-\beta V_{0}(\mathbf{r})} \sum_{k=0}^{N_{\text {states }}} g_{k} e^{-\beta \epsilon_{\mathbf{r}}^{k}}
$$

with $\beta=1 / k_{B} T$ and $g_{k}$ a degeneracy factor, as discussed below. The move is accepted with probability $\min \left(Q(\mathbf{r}) / Q\left(\mathbf{r}^{\prime}\right), 1\right)$. If rejected, the center-of-mass reverts to the position $\mathbf{r}$. The procedure is iterated, to produce a Markov chain of positions weighted by the canonical probability 
density. The Lanzcos rotational state calculations are fairly expensive so we could only perform fairly limited sampling of the different configurations of the center-of-mass of the $\mathrm{H}_{2}$ molecule. Typically our MC runs which determine separation factors (Sec. III) involve only 1000-2000 center-ofmass moves. Monitoring the fluctuations during these calculations, however, as well as repeating calculations for 1000 steps and different initial conditions indicates that the runs effectively jump between several local minimum positions of the $\mathrm{H}_{2}$ center-of-mass in the zeolite many times throughout the calculation, rather than trapping in these local minima. We made no attempt to anneal sufficiently to determine global energy minima within the unit cell. For example, the minimum found at $10 \mathrm{~K}$ and discussed in Sec. IV can only be presumed to be a local minimum, sufficiently deep to trap the molecule at this low temperature.

Degeneracy factors in Eq. (23) arise from nuclear spin; $g_{k}$ is 1 for the spin singlet state, para hydrogen and 3 for the triplet, ortho hydrogen. ${ }^{44}$ The determination of the nuclear spin of state $k$ was made based on the inversion symmetry of the corresponding wave function calculated with the Lanczos algorithm. Because the nuclei of hydrogen are identical fermions, the molecular wave function must be antisymmetric under exchange of the nuclei. The molecular wave function is, in principle, a product of translational, vibrational, electronic, rotational, and nuclear terms, but only the last two are modeled here. If the rotational state $\phi_{\mathbf{r}}^{k}(\theta, \phi)$ is odd (even) under inversion of coordinates (i.e., $\theta \rightarrow \pi-\theta$ and $\phi \rightarrow \phi$ $+\pi)$ then we declare the nuclear spin wave function to be even (odd) under exchange. For example, for free hydrogen, one expects this parity of the rotational level indexed by $J$ to simply vary as $(-1)^{J}$, so that $J$ even (odd) corresponds to para (ortho) hydrogen. A coupling between nuclear spin and rotational state enables the separation of spin isotopes in zeolites and related materials; this is the topic of Sec. III.

\section{POTENTIAL ENERGY SURFACES AND SEPARATION OF SPIN ISOTOPES}

The separation of the spin isotopes, ortho and para hydrogen, was first attempted in the 1950 s by Sandler; ${ }^{45}$ with his most successful separation occurring at $90.2 \mathrm{~K}$ on a $\mathrm{TiO}_{2}$ surface. Some subsequent separation studies involved alumina as the catalytic surface; ${ }^{46,47}$ on this surface, the first complete purifications of ortho hydrogen ${ }^{48,49}$ were achieved. At the heart of the phenomenon of separation is the differential heat of adsorption of ortho and para hydrogen, with the former being more strongly adsorbed. From Sandler onward, people presumed that the preferential adsorption of ortho hydrogen was due primarily to hindered rotation of hydrogen on the adsorbing surface. The so-called separation factor is defined as

$$
S \equiv \frac{\left(Q_{o} / Q_{p}\right)_{\mathrm{ads}}}{\left(Q_{o} / Q_{p}\right)_{\mathrm{gas}}}
$$

where $Q_{o, p}$ are the partition functions of ortho and para hydrogen, respectively, and the numerator and denominator of Eq. (24) refer to the adsorbed and the free, gas phase, respectively. Hindered rotation is relevent because of the correlation between rotational state and nuclear spin statistics. Para hydrogen has a nuclear state with total spin zeroantisymmetric under exchange of the nuclei-so it must exist in a symmetric rotational state; similarly, ortho hydrogen (total nuclear spin 1) exists in an antisymmetric rotational state. ${ }^{44}$ Modifications of these states, so that they need not correspond to free-hydrogen orbitals, are induced by the anisotropic potential near the adsorbing surface. At sufficiently low temperatures, this alters the partition functions in the numerator of Eq. (24), and $S \neq 1$.

Various simple models of the anisotropic potential have been tried, in order to calculate $S$ for comparison with experiment. Potentials which force hydrogen to move as a planar rotor, ${ }^{45,46}$ harmonic potentials which favor either planar rotation or out-of-plane libration, ${ }^{50}$ potentials with both an out-of-plane and an in-plane hindering term, ${ }^{51,52}$ as well as couplings of the above to motions of the center-of-mass normal to the adsorbing surface ${ }^{50,51,53}$ have been explored. All of these models predict the preferential adsorption of ortho hydrogen; at low temperatures, this stems from the fact that all predict a gap between the ground (para) and first excited state(s) (ortho) which is lower than the gap between the $J$ $=0$ and triplet of $J=1$ states for free hydrogen. Typically, in the hindering potential, these two become "tunnel-splitting" states, with an energy gap which is extremely sensitive to the height of the barrier; and the smaller the gap, the larger the numerator in Eq. (24). All of the models mentioned above, save the planar rotor, have adjustable parameters that are specific to the surface, so the best test of any model relies on comparison with data over a wide range of temperatures; experimental data on separation of deuterium (for which para is the preferentially adsorbed species) provides an additional test of the model. ${ }^{50}$ For example, Katorski and White $^{54}$ model hydrogen as a Morse oscillator that is hindered out of plane. They find good agreement for $S$ and heats of adsorption with previous chromatographic experiments involving homo- and heteronuclear diatomics of hydrogen, deuterium, and tritium adsorbed on alumina at $77.4 \mathrm{~K}$. However, experimental data on adsorbtion with leached glass over an (albeit narrow) range of temperatures ${ }^{55}$ shows a systematic deviation from the best fit which the model can provide. As these authors conclude, a less simplified model of the solid surface is probably needed, in which atom-atom interactions are taken into account. Such a model is especially important if a zeolite is chosen as the adsorbent, for it is much less homogeneous than, e.g., alumina. (The first separation on zeolite was performed by Bachmann et al. ${ }^{56}$ in 1962; separations were acheived on both $13 \mathrm{X}$ and zeolite 5A.)

Atom-atom models arose after advances in scientific computing made molecular simulation common. Larin and Parbuzin ${ }^{57}$ improved on the model of a homogeneous zeolite surface; and studied hydrogen and deuterium separation in zeolites 4A (Na-A) and 5A. Interaction energies between host and guest, as well as between guests in the medium coverage limit, were modeled in detail. Ultimately, hindered molecular rotations were still fit to a simple form of the Evett-Sams-MacRury type: 
TABLE III. Separation factors. As is discussed in Sec. IV, an "A" and a " $\mathrm{B}$ " side of a $\mathrm{Na}_{\text {III }}$ ion provide wells in which $\mathrm{H}_{2}$ becomes trapped for the duration of the run.

\begin{tabular}{ccc}
\hline \hline$T(\mathrm{~K})$ & $S$ & $S_{\text {expt }}$ \\
\hline 300 & $1.01 \pm 0.01$ & $\ldots$ \\
152 & $1.04 \pm 0.03$ & 1.058 \\
135 & $1.08 \pm 0.03$ & 1.108 \\
100 & $1.17 \pm 0.06$ & 1.27 \\
50 & $3.4 \pm 0.5^{\mathrm{A}}, 4.5 \pm 0.5^{\mathrm{B}}$ & 2.2 \\
\hline \hline
\end{tabular}

${ }^{\mathrm{a}}$ Experimental data refer to actual or extrapolated results of Gant et al. (Ref. 61 ); see the text for discussion.

$$
V(\theta, \phi)=\lambda \cos ^{2}(\theta)+\mu(1-\cos n \phi)
$$

with $n=2$. This allowed the authors to calculate the rotational contribution to the partition function. Though the study was limited by the authors' ability to consider only a few discrete binding sites and orientations for the guest, they noted good agreement with experiment for a range of low temperatures for low coverage of $4 A$; and were able to correlate the molecular environment of the guest with the shape of the hindering potential. Our model, which accounts only for guest-host interactions, should be sufficient for determining the separation factor for hydrogen at low coverage and temperature. Though it may be the case that such low coverages are difficult to achieve at low temperature, our interest in the single-guest result is supported by Larin and Parbuzin's finding that the interactions between guests at medium coverage are roughly 0.01 times as strong as the guest-host interactions, and that the separation factor depended weakly on coverage (principally because the fields from the $\mathrm{Na}_{\text {III }}$ ion were screened by neighboring $\mathrm{H}_{2}$ molecules). An advantage of our calculation is that hydrogen explores a variety of adsorption sites at thermal equilibrium, as guided by the MC random walk described in Sec. II. We should note though that, as mentioned in Sec. II B, our model neglects vibrational zero point motions of the center of mass. Vibrations against the host walls may be important in weighting Monte Carlo trajectories. Certainly, the difference in vibrational zero-point energy contributes greatly to the separation of mass isotopes like $\mathrm{H}_{2}$ and $\mathrm{D}_{2} \cdot{ }^{50,53,54}$ The calculation is easier if vibrations are assumed to be harmonic, and/or if rotations and vibrations are assumed to be decoupled. For the general case, quantum Monte $\mathrm{Carlo}^{58}$ and path integral ${ }^{59}$ methods are now able to tackle the simultaneous calculation of quantized vibrational and rotation motions. Other methods, including pseudospectral methods, have recently been used in order to understand rovibrations of the water dimer. ${ }^{60}$

To find $S$, the partition functions of Eq. (24) were those of Eq. (23), and were generated during the procedure that determined acceptable steps for the random walk. Thus, at each step, $Q_{o} /\left.Q_{p}\right|_{\text {ads }}$ was determined for a single center-ofmass position within the solid. This quantity was averaged over a 700 step trajectory in order to determine separation factors as they appear in Table III. The listed uncertainties are the standard deviations from the mean values of $S$. Our uncertainties are dominated by the variation of $S$ from place to place in the cavity, rather than by the expected error in a single measurement of $S$. (This is true despite the fact that uncertainties increase as the temperature decreases, and hence the range of positions visited by the hydrogen is reduced. The explanation may lie in the great sensitivity of the tunnel-splitting energy to the height of the hindering barrier.) For comparison with our data, we have listed the experimental results of Gant et al. ${ }^{61}$ Those results, taken during gassolid chromatography experiments in zeolite $\mathrm{Na}$-A, were conducted in the temperature range $135-160 \mathrm{~K}$. We have extrapolated these results where relevent, assuming a linear (Arrhenius) relationship between $\ln (S)$ and $1 / T$. From the experimental data, and on theoretical grounds, one would not expect this relationship to hold over a wide range of temperatures; indeed an extrapolation to $T=300$ yields a nonphysical value of $S<1$, and is not listed in the table. In and near the temperature range of the experiment, agreement with our calculation is very good. These experimental data were also used for comparision in Ref. 57; they had good agreement with experiment, and hence with our calculation, for their model " C.". 62

It is useful to refer atom-atom potential energy surfaces to simple models of the type of Eq. (25). For example, the three favorable adsorption sites in Na-A, according to Ref. 57, were best fit with $\lambda=11.20,10.48,7.07$ and $\mu$ $=5.66,1.29,3.59$. Earlier studies inferred the degree of hindering of the rotor based on experimental measurements of $S$ : MacRury and Sams ${ }^{52}$ fit the leached glass data of Ref. 55 with $\lambda \leqslant 10$ and $\mu \approx 1$. A typical rotational potential energy surface in our study at $100 \mathrm{~K}$ is shown in Fig. 2(a). The surface has two equivalent maxima and minima, separated by saddle points. The height of these saddles above the minima correspond to the height of the barrier to tunneling. For the model of Eq. (25), this saddle height is simply $2 \mu$, as the plot in Fig. 2(b) illustrates. In this model, in-plane hindrance $(\mu \neq 0)$ is synonymous with the existence of a saddle point. It is required in order to obtain small tunnel splittings (that is, for any $\lambda \neq 0$, the tunnel-splitting energy vanishes as $\mu$ grows large), and hence to obtain large separation factors at low temperatures.

Figures 2(a) and 2(b) are cylindrical projections, so for example, the lines $\cos \theta= \pm 1$ correspond to points at the north and south poles of a sphere. The large variations in the potential close to these lines in Fig. 2(b) points out that this model, which originated as an expansion in $\cos \theta$, is not very physical for displacements of the rotor that are far from $\cos \theta=0$. In Ref. 6, we suggest a modification of Eq. (25) for which this unphysical feature is absent. A more natural way to view these potential surfaces is directly on the surface of the sphere. This is done for the data of Fig. 2(a) in Fig. 2(c). From this image, it is clear that the sinusoidal nature of the minimum-energy path between equivalent minima in Fig. 2(a) simply results from the failure of the saddles and other "landmarks" of the potential energy surface to coincide with the reference crystallographic axes $\ldots$ in particular, the $z$ axis from which the polar angle $\theta$ is measured. In fact, the preferred path that the hydrogen bond axis follows, between equivalent minima through a saddle point, lies in a plane.

We analyzed each potential energy surface for the 750 locations (302 unique) of a $50 \mathrm{~K} \mathrm{MC} \mathrm{run,} \mathrm{as} \mathrm{well} \mathrm{as} \mathrm{the} \mathrm{(559}$ 


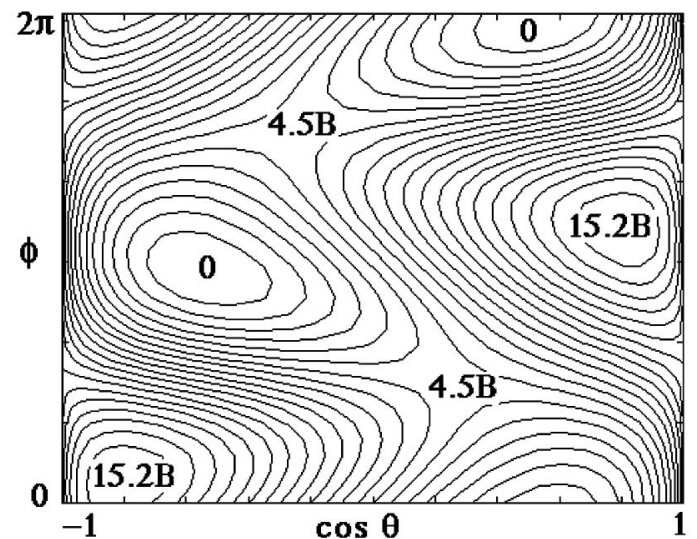

(a)

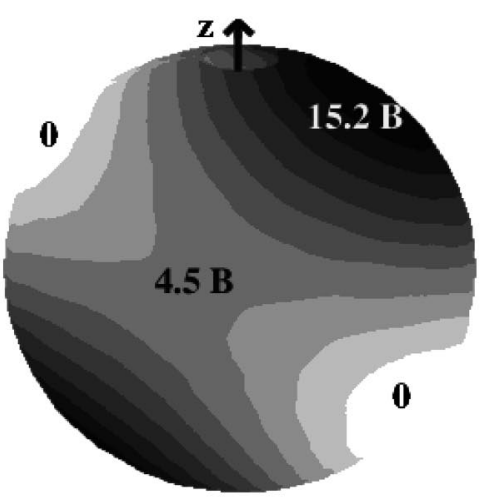

(c)

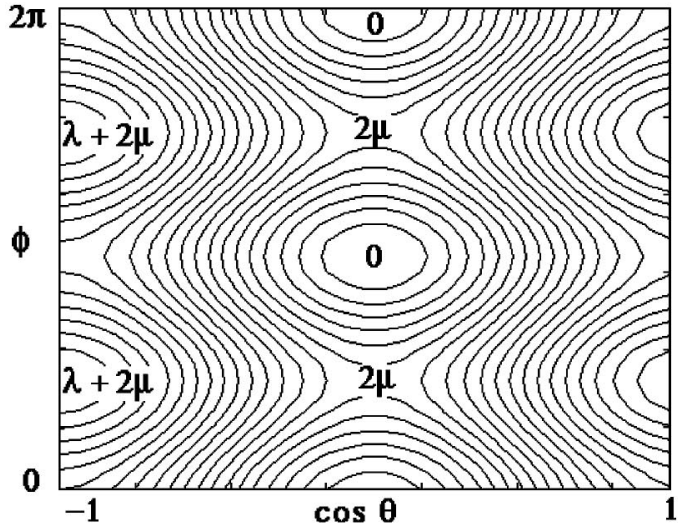

(b)
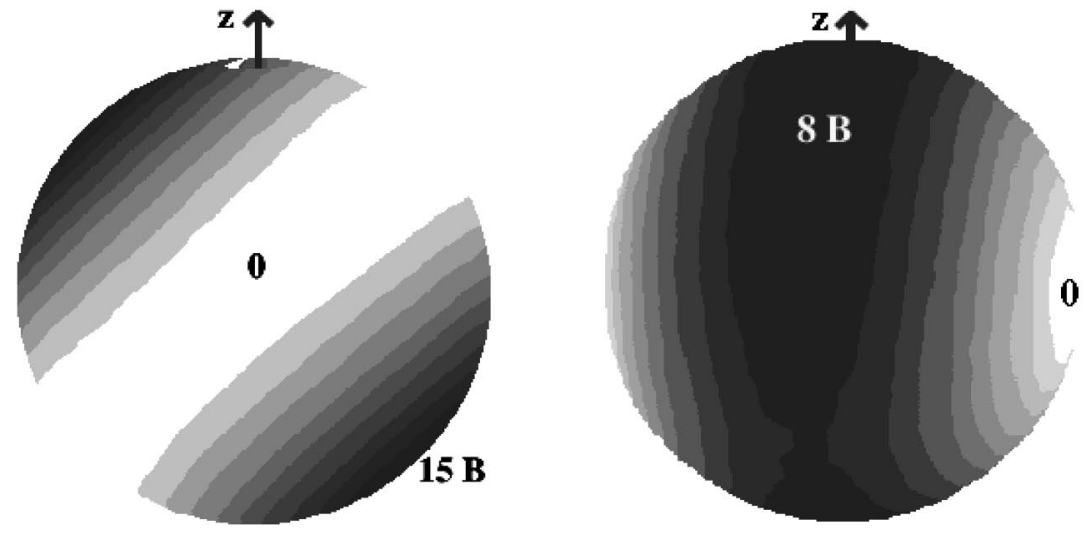

(d)

FIG. 2. (a) Contour map for the part of the potential energy dependent on the orientation of the intramolecular axis. These data correspond to a typical 100 $\mathrm{K}$ location in Na-A. Polar and azimuthal angles are defined in the conventional way, with reference to the $x, y$, and $z$ axes aligned with principal axes of crystal. Here, rotational constant, $B=82 \mathrm{~K}$. (b) Contour map corresponding to Eq. (25). $\lambda$, $\mu$ were chosen as $10.7 B$ and $2.25 B$, so as to produce the same maxima and saddle barrier as in (a). (c) The same data as in (a), but shown as a function of angle on the surface of a sphere. (d) Orientation-dependent potential energy at a $50 \mathrm{~K}$ location in the zeolite in which hydrogen would behave roughly as a "planar rotor." (e) Orientation-dependent potential energy at a $50 \mathrm{~K}$ location in the zeolite in which hydrogen would behave roughly as a "librator."

unique) locations of a $300 \mathrm{~K}$ run, with step sizes of $\delta$ $=0.5 \AA$. This yielded the data of Fig. 3, a histogram of saddle-point barrier heights. The mean barrier height is $4.6 B$ for the case of $50 \mathrm{~K}$, and is slightly smaller, $4.0 B$, at room temperature. Interestingly, the half widths are roughly identical for the two barrier distributions; the standard deviation of each curve is $1.3 B$. The most notable difference is not the half width, but the fact that very low barriers appear in the $300 \mathrm{~K}$ sample, but not at $50 \mathrm{~K}$. Observed barriers varied from approximately $8 B$ down to $1.5 B$ at $50 \mathrm{~K}$, and down to less than $0.5 B$ at $300 \mathrm{~K}$. These end points deserve some comment, because while surfaces of the type of Fig. 2(a) are typical, the end points of the distribution tend to correspond to unusual potential energy surfaces. Figure 2 (d) is a $300 \mathrm{~K}$ surface in which the saddle points are so low that they are roughly isopotential with the minima. In other words, this is the case of a planar rotor, and corresponds to Eq. (25) with $\mu=0$. One would expect such a surface, for example, if hydrogen were adsorbed side-on to a single cation. Reference 57 observes such a surface for hydrogen in zeolite $5 \mathrm{~A}$, in which a single $\mathrm{Ca}$ ion dominates adsorption in this way.
More strange is the case of the $50 \mathrm{~K}$ surface in Fig. 2(e). In this case, it is the maxima that form an equitorial band interrupted by a small saddle, and the hydrogen oscillates in the isolated minima as a librator. The highest barriers in Fig. 3 seem to correspond to surfaces of this type. We saw one position out of more than 1000 examined, in which the potential surface had four local minima. However, these minima were not of equal depth. We must conclude that there were few or no potential environments in our simulation which were qualitatively different from Figs. 2(c)-2(e), and which had a symmetry higher than $n=2$. Though the high symmetry of the aluminosilicate structure of zeolites has caused some authors to speculate that adsorbtion might produce angular potentials with higher symmetries, we have not seen this to be the case. Certainly, the locations of the $\mathrm{Na}$ cations break the symmetry in $\mathrm{Na}-\mathrm{A}$; and interactions with these cations dominate the behavior of adsorbed hydrogen.

The separate terms in expression Eq. (1) can be checked for the importance of their contributions to the potential energy surface. Typically, at locations sampled at $50 \mathrm{~K}$, it is the quadrupole energy $U_{\text {multipole }}$ that varies the most in magni- 

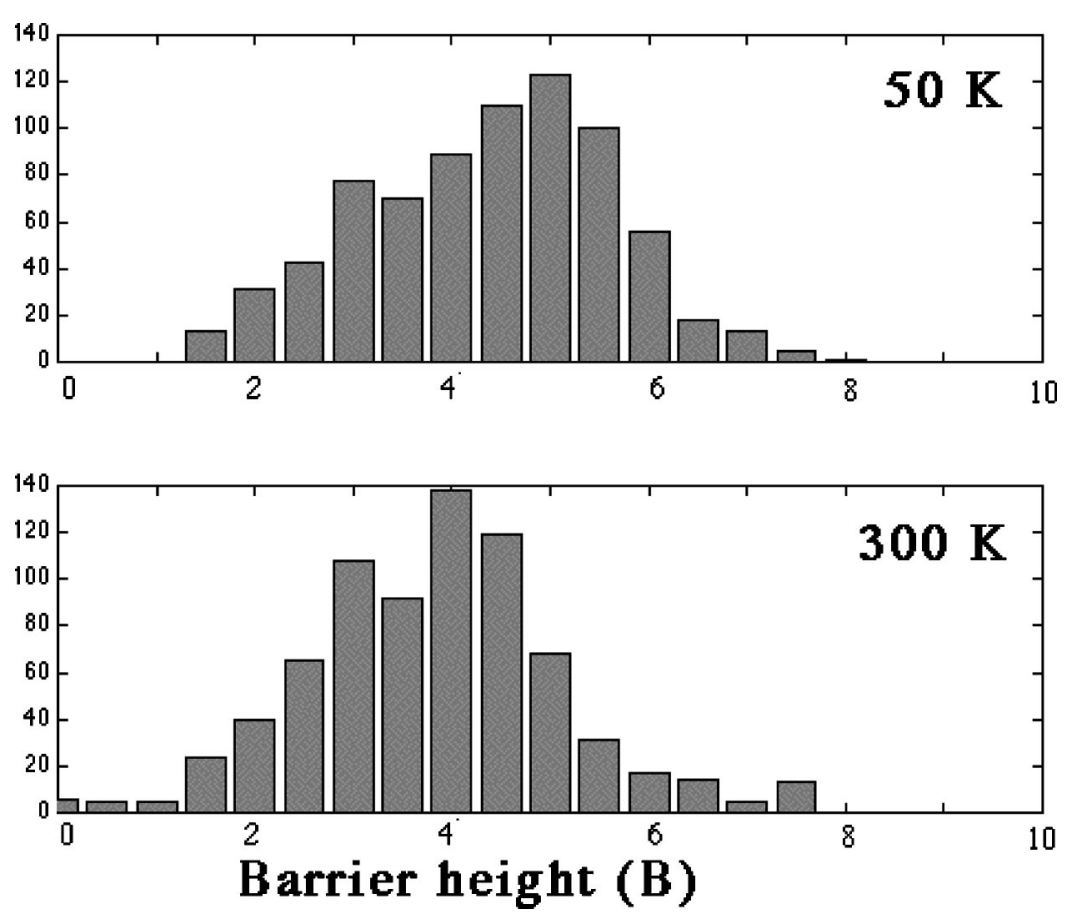

FIG. 3. Histogram of heights of in-plane barriers to rotation for $50 \mathrm{~K}$ (top) and $300 \mathrm{~K}$ (bottom) trajectories. Vertical axes give relative weights; both trajectories consist of 750 configurations. tude, and hence gives the potential energy surface its shape. Further the dominant contribution to this interaction seems to arise from the electrostatic field produced by $\mathrm{Na}$, as opposed to the O, ions, but see Sec. IV and Ref. 6 for a couple of detailed cases, and statistics on the proximity of various $\mathrm{Na}$ ions. Variations in the polarization term, $U_{\text {pol }}$, with angle are generally smaller by a factor of about 4 , and while it induces minor variations in the shape of the potential energy surface, its main effect is to lower the energy uniformly (as does the dispersion attraction). The short-range repulsion raises the energy uniformly; variations in this term are rarely important. (One exceptional case may be when hydrogen is found almost precisely in a doorway.) The importance of a quadrupole interaction with $\mathrm{Na}$ in determining $S$ was also found by Furuyama and Inoue. ${ }^{63}$ Their system, sodium mordenite, has linear channels lined with Na ions. They performed both experimental separations and calculations of the relative importance of the various interaction terms, and concluded that the quadrupole interaction, averaged over positions in the channel, is dominant and produces an average potential barrier of $1.5 \mathrm{kcal} / \mathrm{mol}=8.6 \mathrm{~B}$. Energy level diagrams of Refs. 50 and 51, and the use of only the ground states of ortho and para hydrogen in Eq. (24) then predict values of $S$ in rough agreement with the experiment at 77 and $90 \mathrm{~K}$.

A quadrupole interacting with a single $\mathrm{Na}$ cation would produce a planar rotor potential of the type of Fig. 2(d). If there are two (or more) ions close by the planes of rotation will, in general, cross and produce the generic potential of the type of Fig. 2(c). The librator of Fig. 2(e) arises in an interesting way. The two closest ions are a $\mathrm{Na}_{\text {III }}$ and $\mathrm{Na}_{\mathrm{I}}$ at distances of 2.449 and $2.686 \AA$, respectively. A model containing only these two ions will approximately reproduce the potential energy surface, Fig. 2(e), from the entire crystal. The pair of vectors from hydrogen to each ion very nearly form a right angle. Thus, the planes of rotation defined by quadrupole interaction with each ion cross at almost a right angle. As a result the maxima add to form the "band" across the sphere, as seen in Fig. 2. A configuration in which the two closest ions are $\mathrm{Na}_{\mathrm{III}}$ and $\mathrm{Na}_{\mathrm{I}}$, both roughly $2.5 \AA$ distant, is a typical one; though not the only type found to be adsorptive in our studies. In particular, the minimum-energy configuration found in a series of studies by Larin et al. is of this type; their data will be discussed further in Sec. IV.

In conclusion, at temperatures of $50 \mathrm{~K}$ and above, it is difficult to replace the details of this heterogeneous zeolite surface with a single, simple model, in order to predict $S$. The distribution of saddle barriers of Fig. 3 is wide, and each barrier may or may not be much less than the maximum of the potential energy surface, $(\lambda+2 \mu)$ in the language of Eq. (25). This makes it impossible to propose a single formula that predicts $S$ directly from these data; and an atom-atom simulation seems necessary for comparison with experiment. On the other hand, the simple in- and out-of-plane barrier model is a useful one to keep in mind, together with orderof-magnitude estimates of the model parameters. In Fig. 4 we have plotted the tunnel-splitting energy as a function of the in-plane barrier for the 302 unique steps in a 750-step trajectory simulated at $50 \mathrm{~K}$. These data are to be compared with the predicted dependence of tunnel-splitting energy on the barrier height, $2 \mu$, that appears in Ref. 52. Evidently, out-of-plane barriers in the range of $\lambda=1-10$ yield tunnel splittings which are typical of our simulated results. (It is logical to compare $\lambda+2 \mu$ directly with the difference between the potential maximum and minimum at the various locations in the MC trajectory. For this $50 \mathrm{~K}$ trajectory the difference has a mean of $10.2 B$ and a half-width of $2.5 B$.) These tunnel splittings yield good agreement with experiment; ${ }^{6}$ despite the fact that the typical in-plane barriers are about a factor of 2 smaller than the barrier at the most favorable adsorption site reported in Ref. 57. However, $q_{\mathrm{Na}}$ $=1.0 e$ in this reference, and we noted that our barrier heights increased by a factor of 2 when our model charge was raised 


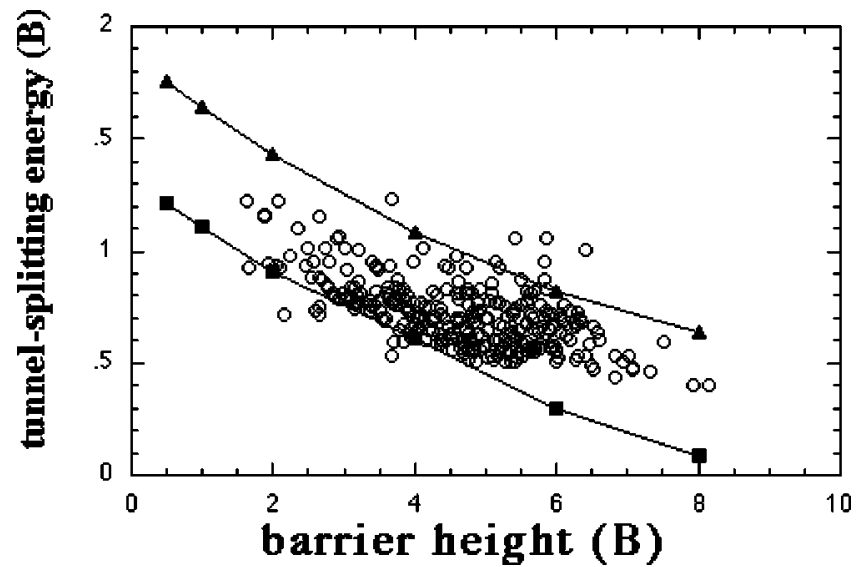

FIG. 4. The difference between ground and first excited state energies (tunnel splitting) vs the in-plane barrier height. Open circles: A 750 step MC trajectory taken at $50 \mathrm{~K}$ (with hydrogen remaining on the " $\mathrm{A}$ " side of $\mathrm{Na}_{\text {III }}$ ), closed triangles: MacRury and Sams (1970) data for $\lambda=1$, solid squares: ditto for $\lambda=10$.

from $q_{\mathrm{Na}}=0.6 e$ to $q_{\mathrm{Na}}=0.8 e .{ }^{6}$ We should also note that the barrier in the Na-Mordenite study of Ref. 63, which is an out-of-plane one, compares favorably in magnitude with the out-of-plane barriers in our study.

One experimental phenomenon on which we have not touched is spin conversion. Chemical and physical mechanisms in real zeolites will convert between ortho and para forms, working against their separation and driving their relative concentrations to the gas phase value. Brown et al. ${ }^{64}$ found that at low tempertures a physical mechanism is dominant: the interaction with paramagnetic ions in the zeolite. The rate of spin conversion has been measured in $4 \mathrm{~A}$ and related systems. ${ }^{64,65}$ In principle, one could therefore predict the degree of conversion, and indeed whether separation could ever be achieved in a particular experiment, given the amount of zeolite surface and the time course of the experimental procedure. In practice, conflicting results ${ }^{61,66}$ are obtained. So, whether a separation is achieved in a given experiment seems to be dependent on the zeolite sample in a way which is difficult to control. ${ }^{67}$ Of course, in our calculation, spin conversion is absent.

\section{PREFERRED LOCATIONS}

A precise knowledge of the location of adsorbate molecules in zeolites is of critical importance for the understanding of interactions with the host, which in turn determine the functionality of such systems for gas storage, separation, and catalysis. While large molecules (such as benzene, for example) can in favorable cases be located by diffraction methods, and the resulting adsorption complex used to derive an interatomic force field, ${ }^{68}$ it is virtually impossible to find very small molecules such as $\mathrm{H}_{2}$ by the same technique. The reason for this is that even at low temperature small molecules may adsorb at a variety of sites, and therefore not contribute to the diffraction pattern in a coherent way. For example, attempts to locate one or two $\mathrm{D}_{2}$ molecules per supercage in CoNa-A and in CaNa-A by neutron diffraction ${ }^{69}$ were indeed unsuccessful.

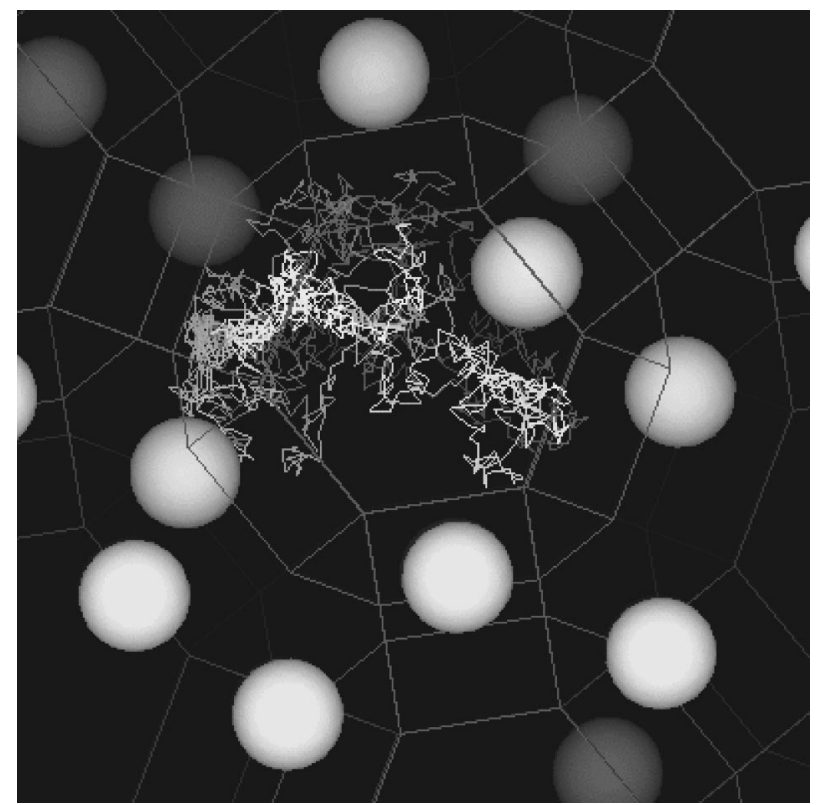

FIG. 5. MC trajectory of length 2000 steps, generated at $T=300 \mathrm{~K}$. White path is the center of mass of the $\mathrm{H}_{2}$ molecule. The $\mathrm{Si} / \mathrm{Al}$ framework and $\mathrm{Na}_{\text {II }}$ and $\mathrm{Na}_{\mathrm{III}}$ ions are shown.

Indirect methods have therefore been used to infer some modest detail about the adsorption sites of $\mathrm{H}_{2}$ in zeolites by IR spectroscopy ${ }^{7,8,16,17,19,70,71}$ or by deducing the transitions of the hindered $\mathrm{H}_{2}$ rotor as described in the present and previous neutron studies. ${ }^{6,72,73}$ Both of these techniques depend on calculations in order to associate the observed excitation with adsorption at a particular site. Therein lies the importance of studies like this one which establish this connection for the hindered rotations of the adsorbed $\mathrm{H}_{2}$ molecule in Na-A. (Some studies which achieve a similar connection for IR vibrational shifts will be cited below.) Moreover, our work illustrates various expected features of $\mathrm{H}_{2}$ binding such as being side-on to the cation because of the predominance of the quadrupole interactions, ${ }^{34,35,63,70,71,74}$ as well as the inaccessibility of the sodalite cage ${ }^{75}$ to $\mathrm{H}_{2}$ at ambient temperatures and low pressures because of steric constraints.

Typical MC trajectories at temperatures of $T=300$ and $50 \mathrm{~K}$ are displayed in Figs. 5 and 6. At $300 \mathrm{~K}$ (Fig. 5), the hydrogen is free to explore the extent of the supercage, and is also free to cross through the octagonal doorways between cages. At $100 \mathrm{~K}$ such crossings are less common during simulation, and hydrogen shows a preference for the viscinity of both a $\mathrm{Na}_{\text {II }}$ and $\mathrm{Na}_{\text {III }}$ ion. At $50 \mathrm{~K}$, the hydrogen has settled into a potential well in this vicinity, and exits from a single well are not observed during any of the $\mathrm{MC}$ trajectories that were generated. (For clarity, $\mathrm{Na}_{\mathrm{I}}, \mathrm{O}$, and framework atoms are not shown in Figs. 5 and 6.)

As mentioned in Sec. II A, a choice was made on a set of sodium locations, and any such choice alters the $P m 3 m$ point-group symmetry of the system. It turns out that for our choice of sodium coordinates, there are distinct favorable binding "sites" for hydrogen on two sides of the $\mathrm{Na}_{\text {III }}$ ion: The role of $\mathrm{Na}_{\text {III }}$ as the favored low temperature adsorption site for $\mathrm{H}_{2}$ in Na-A has been argued in studies by Cohen de Lara and others. ${ }^{21,71,76}$ Figure 6(a) displays a trajectory on 


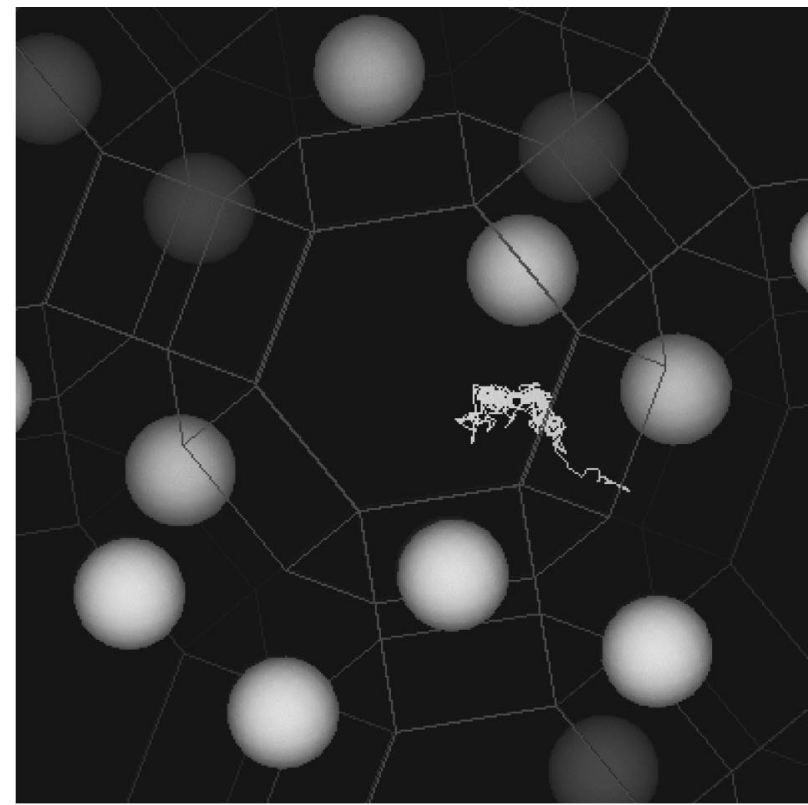

(a)

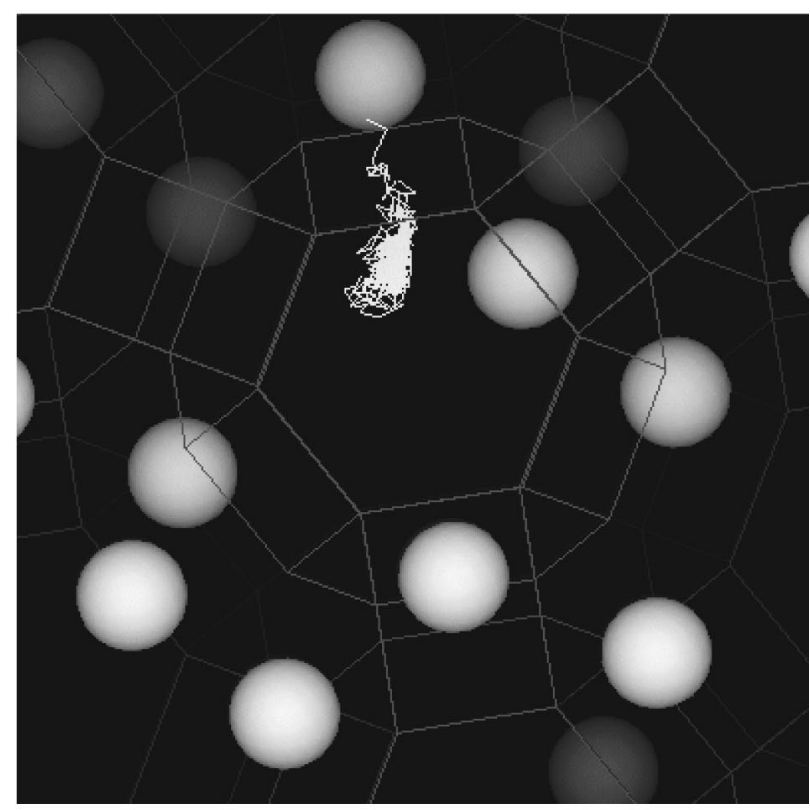

(b)

FIG. 6. Two MC trajectories of length 2000 steps, generated at $T=50 \mathrm{~K}$. (a) Trajectory has been equilibrated on the " $\mathrm{A}$ " side of the $\mathrm{Na}_{\text {III }}$ ion. (b) Trajectory has been equilibrated on the " $\mathrm{B}$ " side of the $\mathrm{Na}_{\mathrm{III}}$ ion.

the so-called " $\mathrm{A}$ " side, in which the $\mathrm{Na}_{\mathrm{II}}-\mathrm{Na}_{\mathrm{III}}$ distance is $3.85 \AA$. (This trajectory produced, for example, the data in Figs. 3 and 4.) The distance is rather larger, $5.11 \AA$, on the "B" side of Fig. 6(b). Figure 7 is a schematic picture of the geometry of $\mathrm{Na}_{\mathrm{II}}$ ion placement on the two sides; a placement dictated by the choice made in Table I. While the potential barrier between these two sites is too high for classi$\mathrm{cal}$, activated transport between them to happen easily at 50 $\mathrm{K}$; the well depths are virtually the same. The average internal energy of hydrogen is $\langle U\rangle=-1370,-1385 \mathrm{~K}$ on the " $A$ " and " $B$ " sides, respectively; with a standard deviation of about $50 \mathrm{~K}$, as one would expect, over a typical MC trajectory.

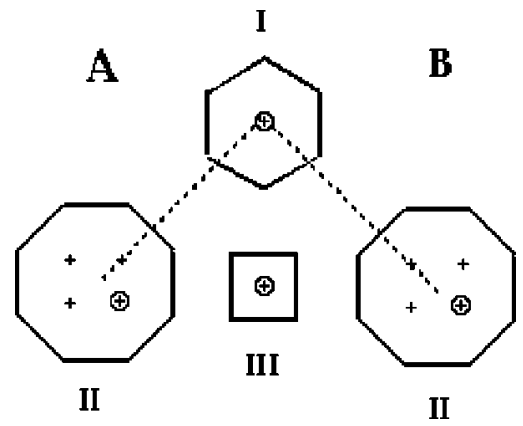

FIG. 7. A schematic view of the geometry near the A and B adsorption "sites" for hydrogen in Na-A. Sodium cations of types I, II, and III are shown as circled plus symbols, and they are located within the appropriate type of framework ring. Two-, three-, and fourfold axes can be imagined as coming out of the page, centered on the appropriate ring. Each dashed line then completes a triangle formed by a fourfold and a threefold axis. The dashed lines can be thought of as the planes, viewed end-on, in which low-temperature hydrogen trajectories lie.

Despite the similar affinity that hydrogen has for these two sites, a disparity in $50 \mathrm{~K}$ separation factors (Table III) is evident. This can be traced to the difference in tunnelsplitting energy which is, on average, around $20 \mathrm{~K}$ lower on the " $\mathrm{B}$ " side. The more "cramped" geometry on the " $\mathrm{A}$ " side of Fig. 7 seems to push hydrogen further from the $\mathrm{Na}_{\mathrm{III}}$. As we will mention below, $50 \mathrm{~K}$ trajectories on the " $\mathrm{A}$ " side seem to prefer the viscinity of $\mathrm{Na}_{\text {II }}$, and a lower rotational barrier results.

As one might expect, not just the tunnel splitting, but all of the low-lying energy level separations are rather different on the two sides. This produces rather different neutron spectra from the two sides, as will be seen in Ref. 6. It is conceivable that in a real zeolite, at low temperatures, the two $\mathrm{Na}_{\mathrm{II}}$ ions of Fig. 7 adjust themselves so that the four cations shown have maximal space between them. This would render the "A" and "B" sides equivalent. (Some recent results on low temperature cationic configurations in Na-A can be found in Ref. 77.) Neutron spectra from a real zeolite Na-A might reflect the fact that hydrogens bound in different wells can make distinctly different contributions to the neutron spectrum.

Though we have not done an exhaustive search for minima and saddle surfaces in this zeolitic potential, Fig. 8 displays hydrogen quenched from $50 \mathrm{~K}$ down to $10 \mathrm{~K}$ on the " $A$ " side. That is, this 2000 step random walk at $10 \mathrm{~K}$ (with $\delta=0.05 \AA$ and an acceptance ratio of $60 \%$ ) was generated beginning with the last position displayed in Fig. 6(a). One finds the hydrogen trapped in close proximity to (around 2.3 $\AA$ away from) a $\mathrm{Na}_{\text {II }}$ ion, near the octagonal doorway. $\mathrm{Na}_{\text {III }}$ and $\mathrm{Na}_{\mathrm{I}}$ are the next-nearest ions, both approximately $4.5 \AA$ distant. The rotational potential has the usual in- and out-ofplane hindering; the tunnel-splitting energy is roughly $44 \mathrm{~K}$ $\left(31 \mathrm{~cm}^{-1}\right)$.

If one neglects the disorder arising from the choice of $\mathrm{Na}_{\text {II }}$ (only one out of four symmetry-equivalent positions can be occupied) and $\mathrm{Na}_{\text {III }}$ (only one out of twelve), then zeolite $\mathrm{Na}-\mathrm{A}$ has various axes of symmetry. These axes can be easily visualized by referring to the ring structure of the $\mathrm{Si} / \mathrm{Al}$ framework: two-, three-, and fourfold axes pass through the 


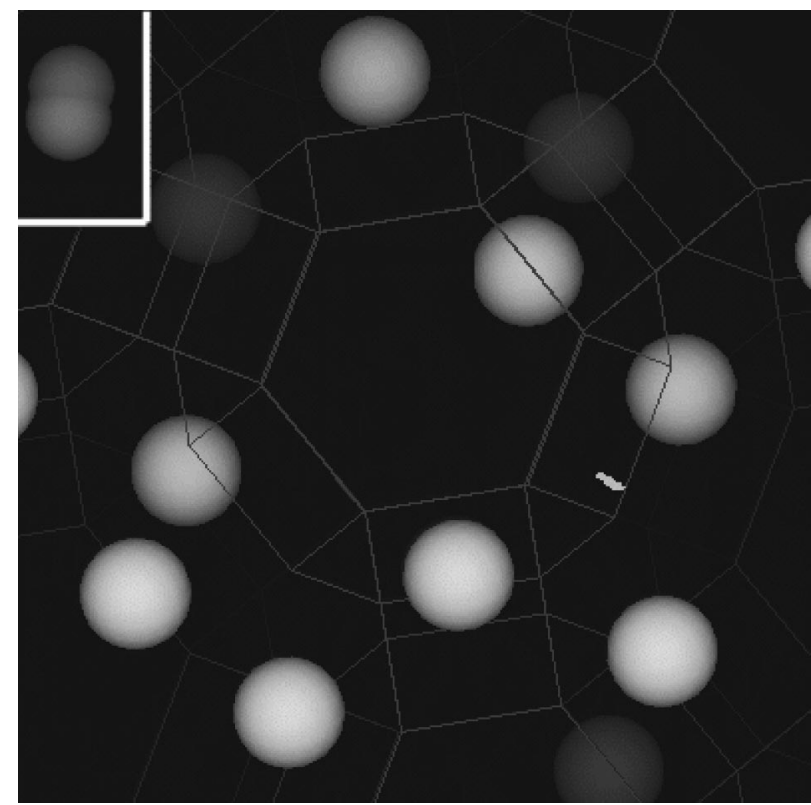

FIG. 8. MC trajectory of length $2000 \mathrm{steps}$, generated at $T=10 \mathrm{~K}$. The center of mass of the $\mathrm{H}_{2}$ molecule is confined to a small region, of extent around $0.2 \AA$, in the vicinity of the $\mathrm{Na}_{\mathrm{II}}$ ion. For reference, the inset shows the size scale of the hydrogen.

centers of square, hexagonal, and octagonal rings, respectively. (See Fig. 3 of Ref. 11.) It is reasonable to postulate that hydrogen minima bear a relationship to, and may even fall upon, a symmetry axis. Symmetry axes are shown in Fig. 9, together with a $100 \mathrm{~K}$ ("A" side) and $50 \mathrm{~K}$ ("B" side) $\mathrm{MC}$ trajectory. While we find that there are two roughly equivalent wells on either side of $\mathrm{Na}_{\mathrm{III}}$, we find that these wells do not seem to coincide with a symmetry axis. Though it is difficult to perceive from a single view as in Fig. 9,

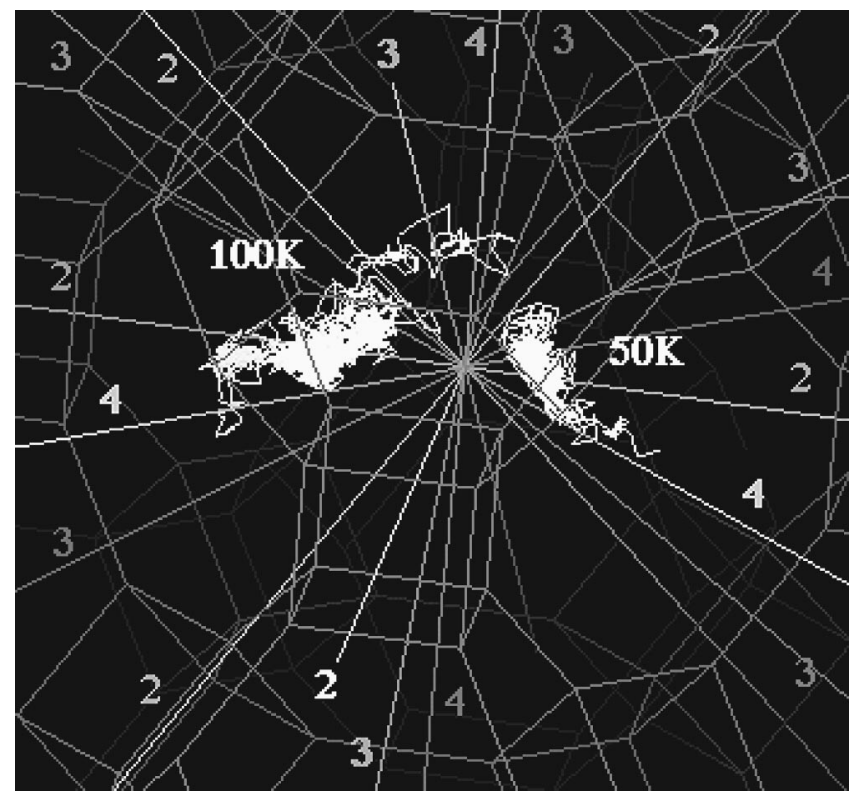

FIG. 9. Two MC trajectories of length 2000 steps, generated at $T=100$ and $50 \mathrm{~K}$. Axes of two-, three-, and fourfold symmetry are labeled. Ions and framework atoms are not shown; a $\mathrm{Na}_{\mathrm{III}}$ ion is situated on the twofold axis closest to the viewer. neither trajectory actually crosses any symmetry axis. Rather, trajectories at $50 \mathrm{~K}$ and below can be characterized as lying in the plane formed by the adjacent three- and fourfold axes. That is, they lie in the plane formed by the vectors from the supercage center to the closest $\mathrm{Na}_{\mathrm{I}}$ and $\mathrm{Na}_{\mathrm{II}}$ ions. Figure 7 is helpful here; the dashed lines can be thought of as coinciding with these planes. With coordinates defined as in Table I, this is the plane which passes through the $z$ axis with a normal direction: $\hat{x}+\hat{y}$ on the " $\mathrm{A}$ " side, and on the " $\mathrm{B}$ ", side it is a plane which passes through the $y$ axis with normal direction: $\hat{x}-\hat{z}$. Thus, the trajectories lie "above" the plane formed by the three- and twofold axes, which is to say, the hydrogen center of mass remains above the plane formed by the vectors to the closest $\mathrm{Na}_{\mathrm{II}}$ and $\mathrm{Na}_{\text {III }}$ ions. Again, Fig. 7 provides a helpful view.

In an interesting set of calculational studies, ${ }^{21,76,78}$ Larin, Cohen De Lara, Leherte and others compute the effect of the $\mathrm{Na}-\mathrm{A}$ environment on the observed IR stretching frequency of $\mathrm{H}_{2}$. (An early study of this type is Ref. 71, with Ref. 79 using this method for $\mathrm{H}_{2}$ in various forms of ZSM-5. Earlier studies of this type for $\mathrm{Na}-\mathrm{Ca}-\mathrm{A}$ were performed in Refs. 19 and 57.) Several important conclusions were drawn, including the best potential model to reproduce the experimental shifts ("ionicity" of the model), ${ }^{21,76}$ the size of the orthopara splitting of the first vibrational transition, and the relative importance of hindered rotations and center-of-mass vibrations to the frequency shift. ${ }^{76}$ In all of the Na-A studies, the two deepest potential wells were found to occur on or near the threefold axes closest to $\mathrm{Na}_{\text {III. }}$. This was also found to be the case for $\mathrm{Ca}-\mathrm{Na}-\mathrm{A}$ according to Ref. 57; while in Ref. 19 one infers from the discussion that the minimum of $-920 \mathrm{~cm}^{-1}$ is near, but not directly on this axis.

Differences in our model of Na-A and that of Larin et al. (crystalline symmetries, slightly different atomic positions, $\mathrm{Na}_{\text {II }}$ and $\mathrm{Na}_{\text {III }}$ disorder, details of the potential) make it unlikely that we would find identical minima. Though the potential model of Larin et al. evolved somewhat in their series of papers, the location of their minimum was consistent. (The authors note that the minimum shifts somewhat when potential parameters are varied.) So it is worth noting that we find a saddle point, but not a global minimum, directly on the $C 3$ axes closest to $\mathrm{Na}_{\mathrm{III}}$; and we would not tend to describe the trajectories of Figs. 6(a) and 6(b) as hovering about this axis. However, consider a typical position on the " $\mathrm{B}$ '" side in our model, in the viscinity of what we must assume is an energy minimum for our model. Such a position has this essential feature in common with the position identified by Larin et al.: It is roughly equidistant from $\mathrm{Na}_{\text {III }}$ and $\mathrm{Na}_{\mathrm{I}}, 2.6$ and $2.7 \AA$, respectively; to be compared with 2.9 and $2.8 \AA$ in Ref. 78. We are also in agreement about the depth of the well; approximately $-1800 \mathrm{~K} .^{21}$ Perhaps it is relevent to examine the statistics of proximity to the important, $\mathrm{Na}_{\mathrm{III}}$ ion. We find that at $50 \mathrm{~K}$ on the " $\mathrm{B}$ " side, $\mathrm{Na}_{\text {III }}$ is the ion closest to $\mathrm{H}_{2}$ more than $75 \%$ of the time, with $\mathrm{Na}_{\mathrm{I}}$ the second closest in these configurations. In the remainder of the configurations, $\mathrm{Na}_{\text {III }}$ is second closest and $\mathrm{Na}_{\mathrm{I}}$ closest.

Interestingly, the situation is different on the " $\mathrm{A}$ " side. During some 2000 step runs at $50 \mathrm{~K}, \mathrm{Na}_{\mathrm{II}}$ is the closest ion on each step, and some configurations involve binding to it at 
a distance of less than $2.5 \AA$, with other ions much more distant. (The $10 \mathrm{~K}$ data discussed below are a configuration of this type.) But some of the lowest energies in these " $\mathrm{A}$ ", trajectories arise when $\mathrm{H}_{2}$ is able to adsorb in a position which is also close to $\mathrm{Na}_{\text {III }}$ (e.g., at a distance of less than 2.9 A).

Of course, in our MC study it is the average energy, including zero-point rotational energy, which guides trajectories. Thus locations where the bare potential energy is low, but zero-point rotational energy is high, may not be weighted very strongly; in contrast to studies that use only the bare potential energy to guide sampling. While we have not analyzed the data exhaustively, a few cases serve to illustrate the situation. For a typical location on the "B'" side, we find that the dispersion attraction, $\mathrm{U}_{\text {disp }}$ and the sum of $U_{\text {rep }}+U_{\text {pol }}$ $+U_{\text {multipole }} \equiv U_{\text {rot }}$ make contributions of roughly equal magnitude to the total potential energy, which is $U=-1886 \mathrm{~K}$. Specifically, $U_{\text {rot }}=-987 \mathrm{~K}$. The zero-point energy in this rotational well is $E_{0}=515 \mathrm{~K}$, which substantially decreases the "effective" depth of the well. In contrast, on the "A'" side, a typical location with $U=-1810 \mathrm{~K}$ has rotational terms which are less important than $U_{\text {disp }}$. Specifically, $U_{\text {rot }}=-462 \mathrm{~K}$, with a zero-point energy of only $E_{0}=99 \mathrm{~K}$. (For what it is worth, we find that the saddle point which seems to correlate with the minimum of Larin et al. has a well depth which is dominated by $U_{\text {rot }}=-859 \mathrm{~K}$, with a significant zero-point rotational energy: $E_{0}=402 \mathrm{~K}$.) Perhaps one can summarize by saying that the two different wells, " $\mathrm{A}$ " and "B," are attractive for different reasons. The former harbors configurations with a large dispersion energy and low rotational barriers, while the latter owes its attractiveness to a deep rotational potential. This issue is significant if one hopes to deduce rotational barriers from the adsorption strength of a site. Unfortunately, it indicates that the two may or may not be correlated - that one might have deep but "flat"' wells, as well as "steep"' wells, in a single host/ guest system.

As was discussed in Sec. III, one might look into the importance of each host atom, and each type of term in Eq. (1), in determining the potential, and hence the binding energy, the orientation of the bond axis, and the ease of rotational tunneling of $\mathrm{H}_{2}$ at various locations in the host. Because our technique involves solving the angular Schrödinger equation on an angular grid, we have additional information about the shape of the rotational wave function of hydrogen, $\Psi_{n}(\boldsymbol{\Omega})$. We will take up this analysis in the sequel to this paper, Ref. 6 .

\section{CONCLUSIONS}

In summary, we have performed a computational study of hydrogen in zeolite $\mathrm{Na}-\mathrm{A}$, in which we have sampled hydrogen at fixed locations in the zeolite cavity (a BornOppenheimer approximation) at temperatures in the range of $10-300 \mathrm{~K}$. Two roughly degenerate adsorption regions are identified, in proximity to $\mathrm{Na}_{\text {III }}$ and/or $\mathrm{Na}_{\mathrm{II}}$ ions. The typical rotational potential energy surface has twofold symmetry, with both in- and out-of-plane hindering. The relationship between cationic positions and potential features is subtle, with both $\mathrm{O}$ and $\mathrm{Na}$ playing a role in determining barrier locations and heights. The distribution of in-plane barriers heights is broad, with a mean between $4 B$ and $5 B$ at temperatures between 50 and $300 \mathrm{~K}$. Tunnel-splitting energies, as determined from a Lanczos solution to the angular Schrödinger equation, are related to these barriers in a way which is consistent with earlier analytical models of MacRury et al. Thermodynamic separation factors for ortho and para hydrogen range from approximately unity at room temperature to $3-5$ at $50 \mathrm{~K}$. These separation factors are in good agreement both with experimental results at intermediate temperatures, and with a previous simulation.

\section{ACKNOWLEDGMENTS}

We are grateful to many individuals for their advice during the course of this study, among them: E. Cohen de Lara, P. Collings, J. E. Fischer, A. B. Harris, L. Xiao, J. Nicol, P. Papanek, R. Pasternack, T. Stephenson, and D. White. We are very grateful for the technical contributions of J. Boccio, G. Martyna, and T. Reese. We would also like to acknowledge the work of the following individuals, who were undergraduate research assistants at Swarthmore College: E. Cheung, E. Engstrom, J. MacKinnon, and S. Pearlman. This research was initiated under the N.S.F. V.P.W. Program No. 9103932, and additionally supported by a Cottrell College Science Award of Research Corporation No. C-3617, and the Faculty Research Support Fund of Swarthmore College. D.F.C. acknowledges NSF support from Grant No. CHE9521793. We thank (the former) N.C.S.A. at the University of Illinois U.C. as well as the Pittsburgh Supercomputing Center, for grants of Supercomputer time.

\section{APPENDIX: BORN-OPPENHEIMER APPROXIMATION}

Consider the following Hamiltonian for the hydrogen molecule:

$$
\begin{aligned}
\hat{H} & =-\frac{\hbar^{2}}{2 m} \nabla^{2}+V_{0}(\mathbf{r})-\frac{\hbar^{2}}{2 I} \hat{L}^{2}+V_{\mathbf{r}}(\boldsymbol{\Omega}) \\
& \equiv \hat{H}_{0}(\mathbf{r})+\hat{H}_{\mathbf{r}}(\boldsymbol{\Omega}) .
\end{aligned}
$$

The first and last two terms constitute $\hat{H}_{0}$ and $\hat{H}_{\mathbf{r}}$, respectively. $\hat{L}$ is the angular momentum operator for a rigid, linear rotor and $I$ is the moment of inertia. $\nabla$ denotes differentiation with respect to the center-of-mass coordinate $\mathbf{r}$. We write the time dependent wavefunction for the $\mathrm{H}_{2}$ rotations and centerof-mass translations as

$$
\psi(\mathbf{r}, \boldsymbol{\Omega}, t)=\sum_{k^{\prime}}\left\langle\boldsymbol{\Omega} \mid k^{\prime} ; \mathbf{r}\right\rangle \chi^{k^{\prime}}(\mathbf{r}, t),
$$

where the rotational state functions, $\left\langle\boldsymbol{\Omega} \mid k^{\prime} ; \mathbf{r}\right\rangle$, satisfy

$$
\left\langle\boldsymbol{\Omega}\left|\hat{H}_{\mathbf{r}}\right| k^{\prime} ; \mathbf{r}\right\rangle=\epsilon_{\mathbf{r}}^{k^{\prime}}\left\langle\boldsymbol{\Omega} \mid k^{\prime} ; \mathbf{r}\right\rangle,
$$

and $\chi^{k^{\prime}}(\mathbf{r}, t)$ are expansion coefficient functions

Substituting Eq. (A2) into $i \hbar(\partial \Psi / \partial t)=\hat{H} \Psi$, it is easily shown that these coefficient functions satisfy the following equations: 


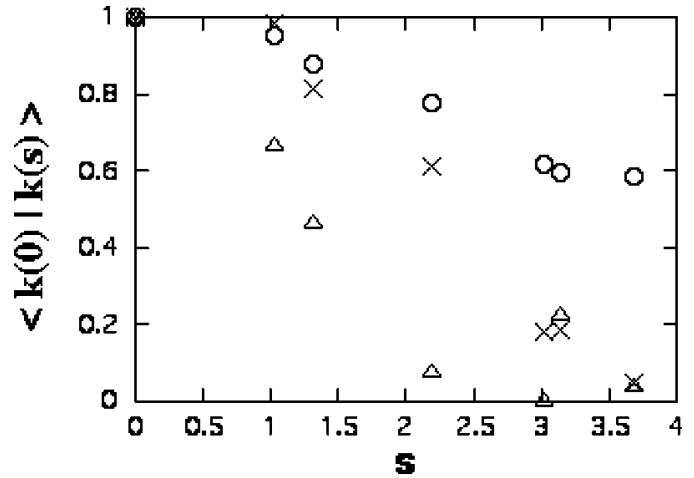

FIG. 10. Overlap of orientational wave functions: $\langle k ; \mathbf{r} \mid k ; \mathbf{r}+\mathbf{s}\rangle . \mathbf{r}$ is a typical location, $(0.85,5.7,0.54) \AA$, on a MC trajectory at $50 \mathrm{~K}$. s points along the trajectory. Open circles: $k=0$, crosses: $k=1$, triangles: $k=2$.

$$
\begin{aligned}
i \hbar \frac{\partial \chi^{k}}{\partial t}= & -\frac{\hbar^{2}}{2 m} \nabla^{2} \chi^{k}+\left[V_{0}(\mathbf{r})+\epsilon_{\mathbf{r}}^{k}\right] \chi^{k}-\frac{\hbar^{2}}{2 m} \\
& \times\left[\sum_{k^{\prime}}\left\langle k ; \mathbf{r}\left|\nabla^{2}\right| k^{\prime} ; \mathbf{r}\right\rangle+\sum_{k^{\prime} \neq k} 2\left\langle k ; \mathbf{r}|\nabla| k^{\prime} ; \mathbf{r}\right\rangle\right. \\
& \cdot \nabla] \chi^{k^{\prime}} .
\end{aligned}
$$

Our rotational Born-Oppenheimer approximation proceeds by assuming that the adiabatic rotational eigenstates $\left|k^{\prime} ; \mathbf{r}\right\rangle$ depend weakly on center-of-mass position $\mathbf{r}$, so the terms in the second line of Eq. (A4) can be ignored compared to the other terms. Under these circumstances, the coefficient functions for the different rotational states decouple, and their dynamics as functions of center-of-mass position are governed by

$$
\hat{H}_{\text {com }}=-\frac{\hbar^{2}}{2 m} \nabla^{2}+V_{0}(\mathbf{r})+\epsilon_{\mathbf{r}}^{k}
$$

In our approach, we assume that this center-of-mass motion can be treated classically. This approximation is particularly vulnerable to breakdown in the case of near-degeneracy of rotational levels. This case is explored in detail in the work of Refs. 42 and 80. In the analogous electronic problem this results in nonadiabatic transitions between electronic states.

In the zeolite, strong anisotropic interactions with the electric field completely break the $2 J+1$-fold degeneracy of hydrogen's rotational energy levels. However, as a numerical check, we examined the variation of the rotational wave function while displacing the center-of-mass by various amounts, taking care to not move into unphysical locations. A successful strategy was to displace the molecule along a MC trajectory. Typical data are given in Fig. 10, in which the self-overlaps of the ground, first, and second excited states are shown as the center of mass is shifted by a distance $s$. One sees a slow variation of orientational wave functions on length scales less than $1 \AA$; the "correlation length" for the variation looks to be between 2 and $3 \AA$.

Further, at several MC-generated locations $\mathbf{r}$, we examined corrections of the dimensionless form: ${ }^{81}$

$$
\begin{aligned}
& \gamma_{1}=\frac{\hbar^{2}}{m\left(\epsilon^{k}-\epsilon^{k^{\prime}}\right)}\left(\left\langle k ; \mathbf{r}|\nabla| k^{\prime} ; \mathbf{r}\right\rangle\right)^{2}, \\
& \gamma_{2}=\frac{\hbar^{2}}{m\left(\boldsymbol{\epsilon}^{k}-\epsilon^{k^{\prime}}\right)}\left\langle k ; \mathbf{r}\left|\nabla^{2}\right| k^{\prime} ; \mathbf{r}\right\rangle .
\end{aligned}
$$

These were evaluated numerically:

$$
\gamma_{1} \approx \frac{\hbar^{2}}{m\left(\epsilon^{k}-\epsilon^{k^{\prime}}\right)}\left(\left\langle k ; \mathbf{r} \mid k^{\prime} ; \mathbf{r}+\mathbf{d} \mathbf{s}\right\rangle / d s\right)^{2}
$$

for $d s$ small; $\gamma_{2}$ was estimated in a similar way. ${ }^{82}$ Test runs examining the four lowest-lying states indicated that the pairings $k=0, k^{\prime}=3$ and $k=1, k^{\prime}=2$ gave the largest values of the matrix elements in Eq. (A6). Taking into account the energy denominator, the latter represented the transition with the largest nonadiabatic character, and typical values of the corrections to the Born-Oppenheimer approximation from this transition were

$$
\gamma_{1} \approx 0.002, \quad \gamma_{2} \approx 0.03 .
$$

Values of these parameters will vary with location in the zeolite, and without having tested all locations, we cannot rule out that we have missed some special sites where "accidental" degeneracy produces a significant nonadiabatic coupling between vibrations and rotations. But based on our testing, it seems that the adiabatic approximation is appropriate.

${ }^{1}$ Zeolites: Science and Technology, edited by F. Ramos Rebeiro et al. (Nijkoff, Boston, 1984).

${ }^{2}$ D. W. Breck, Zeolite Molecular Sieves (Wiley, New York, 1974).

${ }^{3}$ Structure and Reactivity of Modified Zeolites, edited by P. Jacobs (Elsevier, Amsterdam, 1984).

${ }^{4} \mathrm{~K}$. Kalyanasundaram, Photochemistry in Microheterogeneous Systems (Academic, New York, 1987).

${ }^{5}$ J. Weitkamp, M. Fritz, and S. Ernst, in Extended Abstracts of the Ninth International Zeolite Conference, edited by J. B. Higgins, R. Von Ballmoos, and M. M. J. Treacy (Butterworth-Heinemann, London, 1992), p. A27; R. F. Cracknell, P. Gordon, and K. E. Gubbins, J. Phys. Chem. 97, 494 (1993).

${ }^{6}$ J. A. MacKinnon, J. Eckert, D. F. Coker, and A. Bug (in preparation).

${ }^{7}$ A. Zecchina and C. Otero Arean, Chem. Soc. Rev. 25, 187 (1996).

${ }^{8}$ F. Wakabayashi, T. Fujino, J. N. Kondo, K. Domen, and C. Hirose, J. Phys. Chem. 99, 14085 (1995).

${ }^{9}$ T. B. Reed and D. W. Breck, J. Am. Chem. Soc. 78, 5972 (1956)

${ }^{10}$ R. W. G. Wyckoff, Crystal Structures (Wiley, New York, 1968), Vol. 4; V. Subramanian and K. Seff, J. Phys. Chem. 81, 2249 (1977)

${ }^{11}$ J. J. Pluth and J. V. Smith, J. Am. Chem. Soc. 102, 4704 (1980).

${ }^{12}$ A. G. Bezus, M. Kocirik, and E. A. Vasilyeva, Zeolites 7, 327 (1987); P. Santikary and S. Yashonath, J. Chem. Soc., Faraday Trans. 88, 1063 (1992).

${ }^{13}$ E. Cohen de Lara and T. N. Tan, J. Phys. Chem. 80, 1917 (1976); 80, 1923 (1976)

${ }^{14}$ H. Kono and A. Takasaka, J. Phys. Chem. 91, 4044 (1987).

${ }^{15}$ E. Cohen de Lara, R. Kahn, and A. M. Goulay, J. Chem. Phys. 90, 7482 (1989).

${ }^{16}$ O. Zakharieva-Pencheva, H. Bose, H. Forster, W. Frede, and M. Grodzicki, J. Mol. Struct. 122, 101 (1985); A. Sasse and H. Forster, ibid. 349, 97 (1995).

${ }^{17}$ L. M. Kustov and V. B. Kazansky, J. Chem. Soc., Faraday Trans. 87, 2675 (1991); A. Y. Khodakov, L. M. Kustov, V. B. Kazansky, and C. Williams, ibid. 88, 3251 (1992), 90, 3367 (1994); K. Beck, H. Pfeifer, and B. Staudte, ibid. 89, 3995 (1993); S. Bordiga, E. Garrone, C. Lamberti, A. Zecchina, C. O. Arean, V. B. Kazansky, and L. M. Kustov, ibid. 90, 3367 (1994)

${ }^{18}$ J. M. Shin, K. T. No, and M. S. Jhon, J. Phys. Chem. 92, 4533 (1988); S. H. Lee, G. K. Moon, S. G. Choi and H. S. Kim, ibid. 98, 1561 (1994). 
${ }^{19}$ H. Forster, W. Frede, and G. Peters, in Zeolites as Catalysts, Sorbents and Detergent Builders, edited by H. G. Karge and J. Weitkamp (Elseveier, Amsterdam, 1989), p. 545.

${ }^{20}$ K. T. No, J. S. Kim, Y. Y. Huh, W. K. Dim, and M. S. Jhon, J. Phys. Chem. 91, 740 (1987).

${ }^{21}$ A. V. Larin and E. Cohen De Lara, J. Chem. Phys. 101, 8130 (1994).

${ }^{22}$ E. Engstrom, in Proceedings of the Eighth N.C.U.R. Conference on Undergraduate Research, Western Michigan University, Spring, 1994.

${ }^{23}$ N. E. Ghermani, C. Lecomte, and Y. Dusausoy, Phys. Rev. B 53, 5231 (1996-I).

${ }^{24}$ J. O. Hirschfelder, C.F. Curtiss, and R. Byron Bird, Molecular Theory of Gases and Liquids (Wiley, New York, 1965).

${ }^{25}$ A. K. Cheetham and L. M. Bull, Catal. Lett. 13, 267 (1992).

${ }^{26}$ G. C. Maitland, M. Rigby, E. B. Smith and W. A. Wakeham, Intermolecular Forces (Clarendon, Oxford, 1981), Chap. 2.

${ }^{27}$ W. A. Steele, in The International Encyclopedia of Physical Chemistry and Chemical Physics, edited by D. D. Eley and F. C. Tompkins (Pergamon, Oxford, 1974), Vol. 3, Chap. 2.

${ }^{28}$ P. W. Atkins, Physical Chemistry, 4th ed. (Freeman, New York, 1989), Chap. 22.

${ }^{29}$ One must choose the ionization potential, $I$, which appears in the London expression: $A=\frac{3}{2} \alpha_{\mathrm{H}_{2}} \alpha_{k} I_{\mathrm{H}_{2}} I_{k} /\left(I_{\mathrm{H}_{2}}+I_{k}\right)$. We have graphed ionization potential/electronegativity data as in J. E. Huheey, E. A. Keiter, and R. L. Keiter, Inorganic Chemistry, 4th ed. (Harper Collins, New York, 1993), chap. 2. Ionization energies for $\mathrm{O}$ were fit to a quadratic form as suggested in this reference, to arrive at $I$ for $\mathrm{O}^{-0.15}$. Energies for $\mathrm{Na}$ were not amenable to such a fit, and a simple linear interpolation between points was adopted to obtain $I$ for $\mathrm{Na}^{0.6}$.

${ }^{30}$ I. F. Silvera, Rev. Mod. Phys. 52, 393 (1980).

${ }^{31}$ M. F. Falcetta, J. L. Pazun, M. J. Dorko, D. Kitchen, and P. E. Siska, J. Phys. Chem. 97, 1011 (1993).

${ }^{32}$ Several other studies have also fit quantum chemical interactions to choose atom-atom interaction parameters, e.g., that of Ref. 19.

${ }^{33}$ W. L. Dimpfl and B. H. Mahan, J. Chem. Phys. 60, 3238 (1974).

${ }^{34}$ D. A. Dixon, J. L. Gole, and A. Komornicki, J. Phys. Chem. 92, 1378 (1988).

${ }^{35}$ C. W. Bauschlicher, H. Partridge, and S. R. Langhoff, J. Phys. Chem. 96, 2475 (1992).

${ }^{36}$ For comparison, the traditional cutoff for Lennard-Jones potentials is $2.5 \sigma$, which would be approximately $7.5 \AA$, with $\sigma$ the largest interaction diameter between a host $\mathrm{O}$ atom and the guest.

${ }^{37}$ A. K. Nowak, C. J. J. den Ouden, S. D. Pickett, B. Smit, A. K. Cheetham, M. F. M. Post, and J. M. Thomas, J. Phys. Chem. 95, 848 (1991).

${ }^{38}$ P. Demontis, G. B. Suffritti, E. S. Fois, and S. Quartieri, J. Phys. Chem. 96, 1482 (1992).

${ }^{39}$ F. Webster, P. J. Rossky, and R. A. Friesner, Comput. Phys. Commun. 63, 494 (1991); J. K. Cullum and R. A. Willoughby, Lanczos Algorithms for Large, Symmetric Eigenvalue Computations (Birkhauser, Boston, 1985).

${ }^{40}$ G. J. Martyna and B. J. Berne, J. Chem. Phys. 90, 3744 (1989); G. J. Martyna, C. Cheng, and M. L. Klein, ibid. 95, 1318 (1991).

${ }^{41}$ R. F. Curl, Jr., H. P. Hopkins, and K. S. Pitzer, J. Chem. Phys. 48, 4064 (1968).

${ }^{42}$ L. Xiao and D. F. Coker, J. Chem. Phys. 100, 8646 (1994).

${ }^{43}$ M. H. Kalos and P. A. Whitlock, Monte Carlo Methods (Wiley, New York, 1986).

${ }^{44}$ D. A. McQuarrie, Statistical Mechanics (Harper and Row, New York, 1973), pp. 104,105.

${ }^{45}$ Y. L. Sandler, J. Phys. Chem. 58, 58 (1954).

${ }^{46}$ C. M. Cunningham and H. L. Johnson, J. Am. Chem. Soc. 80, 2377 (1958).

${ }^{47}$ E. H. Carter, Jr. and H. A. Smith, J. Phys. Chem. 67, 1512 (1963).

${ }^{48}$ C. M. Cunningham, D. S. Chapin, and H. L. Johnson, J. Am. Chem. Soc. 80, 2382 (1958).

${ }^{49}$ W. R. Moore and H. R. Ward, J. Am. Chem. Soc. 80, 2909 (1958); J. Phys. Chem. 64, 832 (1960)

${ }^{50}$ D. White and E. N. Lassettre, J. Chem. Phys. 32, 72 (1960).

${ }^{51}$ A. A. Evett, J. Chem. Phys. 33, 789 (1960).
${ }^{52}$ T. B. MacRury and J. R. Sams, Mol. Phys. 19, 337 (1970); 20, 57 (1971).

${ }^{53}$ M. P. Freeman and M. J. Hagyard, J. Chem. Phys. 49, 4020 (1968).

${ }^{54}$ A. Katorski and D. White, J. Chem. Phys. 40, 3183 (1964).

${ }^{55}$ M. Mohnke and W. Saffert, in Gas Chromatography, edited by M. van Swaay (Butterworths, London, 1962), p. 216.

${ }^{56}$ L. Bachmann, E. Bechtold, and E. Cremer, J. Catal. 1, 113 (1962).

${ }^{57}$ A. V. Larin and V. S. Parbuzin, Mol. Phys. 77, 869 (1992).

${ }^{58}$ M. Caffarel, P. Claverie, C. Mijoule, J. Andzelm, and D. R. Salahub, J. Chem. Phys. 90, 990 (1989).

${ }^{59}$ D. Marx, S. Sengupta, and P. Nielaba, J. Chem. Phys. 99, 6031 (1993); D. Marx and M. H. Muser, J. Phys.: Condens. Matter 11, R117 (1999).

${ }^{60}$ S. C. Althorpe and D. C. Clary, J. Chem. Phys. 101, 3603 (1994); C. Leforestier, L. B. Braly, K. Lui, M. J. Elrod, and R. J. Saykally, ibid. 106, 8527 (1997); H. Chen, S. Liu, and J. C. Light, ibid. 110, 168 (1999).

${ }^{61}$ P. L. Gant, K. Yang, M. S. Goldstein, M. P. Freeman, and A. I. Weiss, J. Phys. Chem. 74, 1985 (1970).

${ }^{62}$ The models differ on key features (in particular, Ref. 57 includes Si, $\mathrm{Al}$ as explicit centers of force, whereas we "homogenize" Si and Al, interacting effectively through the $\mathrm{O}$ ), so comparison of parameters is not straightforward. Nevertheless, our models agree in terms of the van der Waals radius of O (1.45 $\AA$ ), the ratios of charges of the various oxygens (1:1), and the polarizability of $\mathrm{Na}\left(0.27 \AA^{3}\right.$ as compared with our $\left.0.22 \AA^{3}\right)$. They disagree in the important features of Na radius (one must infer $1.4 \AA$ for theirs vs our $0.98 \AA$ ), charge (their $\left.q_{\mathrm{Na}}=1.0\right)$ and O polarizability $(0.86 \AA$ vs our $1.4 \AA^{3}$ ).

${ }^{63}$ S. Furuyama and H. Inoue, J. Phys. Chem. 87, 1529 (1983).

${ }^{64}$ D. E. Brown, D. D. Eley, and R. Rudham, J. Catal. 16, 292 (1970); 29, 246 (1973).

${ }^{65}$ J. M. Jablonski, Pol. J. Chem. 53, 1103 (1979); R. Stockmeyer, Physica B 180, 508 (1992).

${ }^{66}$ H. Forster and G. Peters, J. Colloid Interface Sci. 126, 260 (1988).

${ }^{67} \mathrm{D}$. White (private communication).

${ }^{68}$ S. M. Auerbach, N. J. Henson, A. K. Cheetham, and H. I. Metiu, J. Phys. Chem. 99, 10600 (1994).

${ }^{69}$ J. Eckert, J. M. Nicol, and T. Vogt (unpublished).

${ }^{70}$ J. Sauer, P. Ugliengo, E. Garrone, and V. R. Saunders, Chem. Rev. 94, 2095 (1994).

${ }^{71}$ E. R. Cohen de Lara, Mol. Phys. 66, 479 (1989).

${ }^{72}$ J. M. Nicol, J. Eckert, and J. Howard, J. Phys. Chem. 92, 7117 (1988).

${ }^{73}$ J. Eckert, J. M. Nicol, J. Howard, and F. R. Trouw, J. Phys. Chem. 100, 10646 (1996).

${ }^{74}$ L. Koubi, M. Blain, E. Cohen de Lara, and J- M. Leclerq, Chem. Phys. Lett. 217, 544 (1994).

${ }^{75}$ D. Fraenkel, J. Chem. Soc., Faraday Trans. 1 77, 2041 (1981).

${ }^{76}$ A. V. Larin and E. Cohen De Lara, Mol. Phys. 88, 1399 (1996).

${ }^{77}$ J. M. Bennett, C. S. Blackwell, and D. E. Cox, ACS Symp. Ser. 214, 143 (1983).

${ }^{78}$ A. V. Larin, F. Jousse, L. Leherte, and D. P. Vercauteren, Chem. Phys. Lett. 274, 345 (1997).

${ }^{79}$ T. Yamazaki, T. Watanuki, S. Ozawa, and Y. Ogino, Mol. Phys. 73, 649 (1991).

${ }^{80}$ L. Xiao and D. F. Coker, J. Chem. Phys. 102, 1107 (1995); H. S. Mei, L. Xiao, and D. F. Coker, ibid. 105, 3938 (1996).

${ }^{81}$ A. Brooks Harris (unpublished).

${ }^{82}$ Equation (9b) of M. Bixon and J. Jortner, J. Chem. Phys. 48, 715 (1968).

${ }^{83}$ J. Applequist, J. R. Carl, and K. K. Fung, J. Chem. Soc. 94, 2953 (1972).

${ }^{84}$ U. Buck, F. Huisken, A. Kohlhase, D. Otten, and J. Schaefer, J. Chem. Phys. 78, 4439 (1983)

${ }^{85}$ P. R. van Tassel, H. T. Davis, and A. V. McCormick, Mol. Phys. 73, 1107 (1991).

${ }^{86}$ R. Kahn, E. Cohen de Lara, and E. Viennet, J. Chem. Phys. 91, 5097 (1989).

${ }^{87}$ A. V. Kiselev, A. A. Lopatkin, and A. A. Shulga, Zeolites 5, 261 (1985).

${ }^{88}$ A. G. Bezus, M. Kocirik, and A. A. Lopatkin, Zeolites 4, 346 (1984).

${ }^{89}$ Handbook of Chemistry and Physics, 55th ed. (CRC Press, Boca Raton, FL, 1975). 